CU-TP-662 and RU-95-2-B

\title{
NONCOMPACT LATTICE FORMULATION OF GAUGE THEORIES
}

\author{
R. Friedberg, T. D. Lee \\ Columbia University, New York, N.Y. 10027 \\ Y. Pang \\ Columbia University, New York, N.Y. 10027 \\ and Brookhaven National Laboratory, Upton, N.Y. 11973 \\ H. C. Ren \\ The Rockefeller University, New York, N.Y. 10021
}

\begin{abstract}
We expand the gauge field in terms of a suitably constructed complete set of Bloch wave functions, each labeled by a band designation $n$ and a wave number $\vec{K}$ restricted to the Brillouin zone. A noncompact formulation of lattice QCD (or QED) can be derived by restricting the expansion only to the $0^{t h}$-band ( $n=0)$ functions, which are simple continuum interpolations of discrete values associated with sites or links on a lattice. The exact continuum theory can be reached through the inclusion of all $n=0$ and $n \neq 0$ bands, without requiring the lattice size $\ell \rightarrow 0$. This makes it possible, at a nonzero $\ell$, for the lattice coupling $g_{\ell}$ to act as the renormalized continuum coupling. All physical results in the continuum are, of course, independent of $\ell$.
\end{abstract}

This research was supported in part by the U.S. Department of Energy. 


\section{INTRODUCTION}

In a recent paper $^{1}$ (hereafter referred to as I) we proposed a new lattice formulation of the continuum field theory. Instead of the usual Fourier series, the continuum field operators are expanded in terms of a suitably chosen complete set of orthonormal Bloch functions

$$
\left\{f_{n}(\vec{K} \mid \vec{r})\right\}
$$

where $\vec{K}$ denotes the Bloch wave number restricted to the Brillouin zone, and $n$ labels the different bands, similar to the one-particle wave functions in a crystal (i.e., $e^{-i \vec{K} \cdot \vec{r}} f_{n}(\vec{K} \mid \vec{r})$ has the periodicity of the lattice). The lattice approximation is then derived by either restricting it to only one band (say, $n=$ 0 ), or to a few appropriately defined low-lying bands. Since the inclusion of all bands is the original continuum problem, there is a natural connection between the lattice and the continuum in this method. By including the contributions due to more and more bands, one can systematically arrive at the exact continuum solution from the lattice approximation. There is a large degree of freedom in choosing the Bloch functions (1.1), as the original continuum theory has no crystal structure. These extra degrees of freedom are analogous to gauge fixing; the final answer to the continuum problem is independent of the particular choice of Bloch functions (or the lattice structure).

In paper I we gave a few simple examples of such functions and applied them to spin- 0 and spin- $\frac{1}{2}$ fields. We noted that this approach provides an effective means of removing the spurious lattice fermion solutions for the Dirac equation. The underlying reason is quite simple: After all, in any crystal in nature all the electrons do move in a lattice and satisfy the Dirac equation; yet there is not a single physical result that has ever been entangled with a spurious fermion solution. Consequently, it should not be that difficult to get rid of these unphysical elements.

On a discrete lattice, particles hop from point to point, whereas in a real crystal the lattice structure is embedded in a continuum and electrons move continuously from lattice cell to lattice cell. In a discrete system, the lattice functions are defined only on individual points (or links, as in the case of the gauge field). However, in a crystal, each Bloch wave $f_{n}(\vec{K} \mid \vec{r})$ is a continuous function in $\vec{r}$, and herein lies one of the essential differences. In the case of gauge theories, there is an additional complication, since the usual lattice 
gauge action ${ }^{2,3}$ is compact, which makes it intrinsically different from the noncompact continuum action (except in the limit of weak coupling or infinitesimal lattice size). As we shall see, these differences can be resolved by extending the lattice formulation developed in I to both quantum electrodynamics (QED) and quantum chromodynamics (QCD). The result is a new noncompact lattice formulation of gauge theories that can serve as the first approximation of the continuum theory in a systematic way, without requiring the lattice size $\ell \rightarrow 0$.

The freedom in choosing any complete set of Bloch functions allows us to explore a family of new functions that are convenient to use. For higher bands, we would like the Bloch functions to stay very close to the Fourier series of high wave numbers, so that renormalization calculations can be carried out analytically. On the other hand, the zeroth band functions $f_{0}(\vec{K} \mid \vec{r})$ should be made of simple continuum interpolations of the discrete values given at lattice (or link) sites; this way, the restriction to zeroth band would naturally resemble the usual lattice theory. Furthermore, in order to retain the "locality" character of the original continuum theory in the one-band approximation, we require $f_{0}(\vec{K} \mid \vec{r})$ to satisfy, for any lattice site $j$ located at $\vec{r}_{j}$,

$$
\frac{1}{\sqrt{\Omega}} \sum_{\vec{K}} e^{-i \vec{K} \cdot \vec{r}_{j}} f_{0}(\vec{K} \mid \vec{r})=0 \quad \text { if } \quad\left|\vec{r}-\vec{r}_{j}\right|>\nu \ell
$$

with $\nu$ a finite number $O(1), \ell$ the unit lattice size, $\Omega$ the total volume $>>\ell^{3}$ and $\vec{K}$ being summed over all wave numbers within the Brillouin zone. This lattice locality condition rules out the simple Fourier series for $f_{0}(\vec{K} \mid \vec{r})$, since for $f_{0}(\vec{K} \mid \vec{r})=\Omega^{-\frac{1}{2}} e^{i \vec{K} \cdot \vec{r}}$ the corresponding sum (1.2) for a cubic lattice with $\Omega=L^{3}$ would be

$$
\frac{\sin \frac{\pi}{\ell}\left(x-x_{j}\right) \sin \frac{\pi}{\ell}\left(y-y_{j}\right) \sin \frac{\pi}{\ell}\left(z-z_{j}\right)}{L^{3} \sin \frac{\pi}{L}\left(x-x_{j}\right) \sin \frac{\pi}{L}\left(y-y_{j}\right) \sin \frac{\pi}{L}\left(z-z_{j}\right)}
$$

which is non-local. Instead, condition (1.2) leads to the concept of "lump functions" and "link functions", which will be introduced in Section 2.

For applications to gauge theories, there is also the problem of compatibility between the continuum gauge-fixing condition and the band decomposition. We discuss QED first in the time-axial gauge in Section 3.1 and then in the Coulomb gauge in Section 3.2. By using suitable combinations of these lump and link functions we show that in both cases the continuum gauge requirement, 


$$
A_{4}(\vec{r}, t)=0
$$

for the time-axial gauge or

$$
\vec{\nabla} \cdot \vec{A}(\vec{r}, t)=0
$$

for the Coulomb gauge, can hold within each $n^{\text {th }}$ band at all $\vec{r}$ and $t$, where $A_{\mu}(\vec{r}, t)$ is the electromagnetic field operator. Thus, as we shall see, in the lattice approximation (i.e., with $A_{\mu}(\vec{r}, t)$ restricted only to the $n=0$ band), we have the interesting situation of having the continuum gauge-fixing equation as well as its discrete realization. For example, in the Coulomb gauge and with the restriction to $0^{t h}$ band, $\vec{A}(\vec{r}, t)$ is a simple continuum interpolation of its discrete values $a_{i j}$, each of which is associated with a link $\ell_{i j}$, where $\ell_{i j}$ connects the $i^{\text {th }}$ lattice site to its nearest neighboring site $j$. The discrete version of $\vec{\nabla} \cdot \vec{A}=0$ refers to Kirchoff's law

$$
\sum_{j} a_{i j}=0
$$

which is valid at every $i$, with $j$ summed over all its nearest neighbors. As will be shown by (3.37)-(3.39), (4.10) and (4.14)-(4.15) below, in our formulation the discrete Kirchoff law implies the validity of the continuum $\vec{\nabla} \cdot \vec{A}=0$ within the one-band approximation, and vice versa. Such equivalent formulas can be further generalized. Take $\vec{\nabla} \cdot \vec{A}=0$ : in the continuum, this implies that $\vec{A}$ equals the curl of another vector function, say $\vec{A}=\vec{\nabla} \times \vec{I}$, and therefore $\vec{A}$ becomes invariant under the transformation $\vec{I} \rightarrow \vec{I}+\vec{\nabla} \chi$. We are pleasantly surprised to find that, within the same one-band approximation in the Coulomb gauge, each of these continuum equations has its exact equivalent discrete form (see (4.24)-(4.29) below), giving rise to a family of relations between the continuum and the discrete. Of course, a change in the gauge has to induce a change in the corresponding Bloch functions, as will be illustrated in Section 3 for QED.

Similar considerations are also applicable to a generalized Coulomb-like gauge where, instead of $\vec{\nabla} \cdot \vec{A}=0$, we have at all continuum $\vec{r}$

$$
\int\left(\vec{r}|\vec{\Gamma}| \vec{r}^{\prime}\right) \cdot \vec{A}\left(\vec{r}^{\prime}\right) d^{3} r^{\prime}=0
$$

with $\vec{\Gamma}$ a linear operator independent of $A_{\mu}(\vec{r}, t)$. While QED is a relatively trivial model, the analysis given in Section 3 provides the necessary tools for the noncompact lattice formulation of non-Abelian theories. 
The extension to QCD in the Coulomb or Coulomb-like gauge is straightforward since the gauge condition, (1.5) or (1.7), is linear and there are no additional supplementary conditions; hence, the group index is external to the band decomposition. The details are given in Section 4. Because the noncompact lattice QCD formulation is the $0^{t h}$-band approximation of the continuum $\mathrm{QCD}$, it is possible to regard the lattice QCD coupling constant $g_{\ell}$ as the exact renormalized coupling constant of continuum $\mathrm{QCD}$, without requiring the lattice size $\ell \rightarrow 0$ (i.e., at a finite $\ell^{-1}$ ), as will be studied in Section 5. We discuss how this approach is related to the conventional renormalization procedure in continuum QCD. By taking advantage of the asymptotic freedom of the theory, we show that, in terms of the perturbation series in the Coulomb gauge, the bare coupling $g_{0}$ and the conventional renormalized coupling $g_{R}$ of continuum QCD can be readily expressed in terms of the lattice coupling $g_{\ell}$ in a systematic way.

The extension to QCD in the time-axial gauge is more complicated: While the gauge condition $A_{4}(\vec{r}, t)=0$ being a linear equation offers no problem in the band decomposition, the generalization of the supplementary "Gauss Law" condition on the state vector ((3.19) below) becomes nonlinear in QCD, which means that in solving this constraint, there would be additional coupling between different bands which must be taken into account. The details will not be analysed in this paper. 


\section{MATHEMATICAL PRELIMINARIES}

In this section, we consider only the one-space-dimensional case.

\subsection{Lump Functions}

These functions are defined by

$$
L_{m}(x) \equiv \int_{-\infty}^{\infty} \frac{d k}{2 \pi} \frac{\left(2 \sin \frac{k}{2}\right)^{m}}{k^{m}} \times \begin{cases}e^{i k x / \ell} & \text { for } m \text { even } \\ e^{i k\left(x-\frac{\ell}{2}\right) / \ell} & \text { for } m \text { odd }\end{cases}
$$

where $m$ is a positive integer $>0$ and $x$ is the continuous coordinate variable. (When $m=0, L_{m}(x)$ becomes the $\delta$-function, which does not serve our particular purpose.) As we shall see, these functions are nonzero (shaped like a lump) only within $m$ lattice cells, each of length $\ell$. When $m$ is even and $|x|>\frac{m}{2} \ell$, we may deform the integration path in the complex $k$-plane into a semi-circle of infinite radius encompassing the upper, or lower, half-plane depending on whether $x$ is greater than $\frac{m}{2} \ell$, or less than $-\frac{m}{2} \ell$; this leads to zero for (2.1). Likewise, when $m$ is odd the integral is also zero for $\left|x-\frac{\ell}{2}\right|>\frac{m}{2} \ell$. In the next section, by using these lump functions we can construct Bloch wave functions that satisfy the lattice locality condition (1.2). From the definition, we see that for $m$ even

$$
\ell \frac{d L_{m}(x)}{d x}=-L_{m-1}(x)+L_{m-1}(x+\ell)
$$

and for $m$ odd

$$
\ell \frac{d L_{m}(x)}{d x}=L_{m-1}(x)-L_{m-1}(x-\ell) .
$$

The lump functions of lower order, $m=1,2$ and 3 , are particularly useful in later sections. Because, in the application to gauge theories, there will be several different kinds of indices, it is convenient to give these functions special names. We introduce

$$
\begin{gathered}
C(x) \equiv L_{1}(x)= \begin{cases}1 & \text { for } 0<x<\ell \\
0 & \text { otherwise }\end{cases} \\
\Delta(x) \equiv L_{2}(x)= \begin{cases}1-\left|\frac{x}{\ell}\right| & \text { for }|x|<\ell \\
0 & \text { otherwise }\end{cases}
\end{gathered}
$$

and

$$
S(x) \equiv L_{3}(x)= \begin{cases}\frac{1}{8}\left[\left(3-2\left|\frac{x}{\ell}-\frac{1}{2}\right|\right)^{2}-3\left(1-2\left|\frac{x}{\ell}-\frac{1}{2}\right|\right)^{2}\right] & \text { for }\left|x-\frac{\ell}{2}\right|<\frac{\ell}{2} \\ \frac{1}{8}\left(3-2\left|\frac{\ell}{\ell}-\frac{1}{2}\right|\right)^{2} & \text { for } \frac{\ell}{2}<\left|x-\frac{\ell}{2}\right|<\frac{3 \ell}{2} \\ 0 & \text { otherwise }\end{cases}
$$


Here, the notation $C(x)$ is chosen for its being a constant within $0<x<\ell$, $\Delta(x)$ for being like a triangle within $-\ell<x<\ell$ and $\mathcal{S}(x)$ for its nonzero part being a second order polynomial in $x$ (within unit intervals of $\ell$ ).

In general, the nonzero parts of all $L_{m}(x)$ are positive polynomials of $(m-$ $1)^{t h}$ order within each $\ell$-interval. For $m>1, L_{m}(x)$ is continuous at all $x$. It follows from $(2.2)-(2.3)$ that for $m>2$ all $d L_{m}(x) / d x$ are continuous functions; for $m>3$, all $d^{2} L_{m}(x) / d x^{2}$ are continuous, etc. When $m$ is even $>0, L_{m}(x)=L_{m}(-x)$; it peaks at $x=0$, decreases monotonically to zero at $|x|=\frac{m}{2} \ell$ and remains zero for $|x|>\frac{m}{2} \ell$. When $m$ is odd $>1, L_{m}\left(x-\frac{\ell}{2}\right)=$ $L_{m}\left(-x+\frac{\ell}{2}\right)$; its maximum is at $x=\frac{\ell}{2}$, decreases monotonically to zero at $\left|x-\frac{\ell}{2}\right|=\frac{m}{2} \ell$ and remains zero for $\left|x-\frac{\ell}{2}\right|>\frac{m}{2} \ell$.

Another useful property is, within the range $0<x<\ell$,

$$
\sum_{j=-(m-2) / 2}^{m / 2} L_{m}(x-j \ell)=\text { constant } \quad \text { for } \quad m \text { even }
$$

and

$$
\sum_{j=-(m-1) / 2}^{(m-1) / 2} L_{m}(x-j \ell)=\text { constant for } m \text { odd }
$$

which can be readily proven by noting that their derivatives are both zero, on account of $(2.2)-(2.3)$.

\subsection{Bloch Functions}

Given an $m$, the corresponding (unnormalized) $0^{\text {th }}$ band Bloch function is given by

$$
\mathcal{F}(K \mid x)=\sum_{j=-\infty}^{\infty} e^{i j \theta} L_{m}(x-j \ell)
$$

with

$$
\theta=K \ell
$$

which satisfies

$$
|\theta| \leq \pi
$$

Because

$$
\mathcal{F}(K \mid x+\ell)=e^{i \theta} \mathcal{F}(K \mid x)
$$


the passage from an infinite lattice to a finite one of size $N \ell$ and with the periodic boundary condition can be readily derived by requiring

$$
K N \ell=2 \pi \times \text { integer } .
$$

This convention will be used subsequently. It follows from (2.5) and (2.6) that, at $K=0, \mathcal{F}(K \mid x)$ is a constant at all $x$. Furthermore, the sum $N^{-1} \sum_{K} e^{-i K j \ell}$ $\times \mathcal{F}(K \mid x)=L_{m}(x-j \ell)$ satisfies the lattice locality condition (1.2).

To construct the higher-band Bloch functions, we follow one of the simple procedures given in I. Let

$$
\psi_{p}(x)=(N \ell)^{-\frac{1}{2}} e^{i p x}
$$

be the usual plane-wave functions with $p N \ell=2 \pi \times$ any integer. Resolve

$$
p=K+\frac{2 \pi n}{\ell}
$$

where $n=\cdot \cdot,-2,-1,0,1,2, \cdot \cdot$. At a $K$ given by (2.8)-(2.9), write (2.12) as

$$
\psi_{n}(x)=(N \ell)^{-\frac{1}{2}} e^{i K x} e^{i 2 \pi n x / \ell} .
$$

The complete set of orthonormal Bloch functions, given by $\left\{f_{n}(K \mid x)\right\}$, can be obtained by requiring, for $n=0$

$$
f_{0}(K \mid x)=c_{m} \mathcal{F}(K \mid x),
$$

with $c_{m}$ the normalization constant, and for $n \neq 0$

$$
f_{n}(K \mid x)=\psi_{n}(x)-\frac{f_{0}(K \mid x)+\psi_{0}(x)}{1+a_{0}} a_{n}
$$

where (for all $n$, including 0 )

$$
a_{n} \equiv \int_{0}^{N \ell} f_{0}(K \mid x)^{*} \psi_{n}(x) d x
$$

and ${ }^{*}$ the complex conjugation. It follows then

$$
\int_{0}^{N \ell} f_{n}(K \mid x)^{*} f_{n^{\prime}}\left(K^{\prime} \mid x\right) d x=\delta_{K K^{\prime}} \delta_{n n^{\prime}}
$$


where $\delta_{K K^{\prime}}$ and $\delta_{n n^{\prime}}$ are Kronecker symbols. By using (2.1) and (2.7) we see that

$$
\left|a_{n}\right|^{2}=\left|c_{m}\right|^{2} \frac{2^{m}(1-\cos \theta)^{m}}{(\theta+2 \pi n)^{2 m}}
$$

Since $\sum_{n=-\infty}^{\infty}\left|a_{n}\right|^{2}=1$, we may write (2.19) as

$$
\left|a_{n}\right|^{2}=(\theta+2 \pi n)^{-2 m}\left[\sum_{n^{\prime}=-\infty}^{\infty}\left(\theta+2 \pi n^{\prime}\right)^{-2 m}\right]^{-1} .
$$

Thus, when $\theta \rightarrow 0$,

$$
\left|a_{0}\right|^{2} \rightarrow 1-2 \theta^{2 m} \sum_{n=1}^{\infty}(2 \pi n)^{-2 m}=1-\frac{\theta^{2 m} B_{m}}{(2 m) !}
$$

and, for $n \neq 0,\left|a_{n}\right|^{2} \rightarrow \theta^{2 m}(2 \pi n)^{-2 m}$ where $B_{m}$ denotes the Bernoulli numbers with $B_{1}=\frac{1}{6}, B_{2}=\frac{1}{30}, B_{3}=\frac{1}{42}$, etc.

We note that, given $\mathcal{F}(K \mid x)$, there are many equally simple ways to construct the complete set of Bloch functions, as discussed in I.

\section{Remarks:}

1. When $m=0, L_{m}(x)$ becomes $\delta(x / \ell)$, as mentioned before; therefore, the expansion (2.7) reduces to the usual expression for a lattice of discrete points.

2. In (2.19), we see that for nonzero $n$ and $m$ the factor $(\theta+2 \pi n)^{-2 m}$ is always less than $[(2|n|-1) \pi]^{-2 m}$. In $D$-dimension, (2.19) will be changed into a product of $D$ such factors, one for each space dimension. Thus, the higher band Bloch functions are very close to the corresponding Fourier series of high wave numbers (see, e.g., Eq. (3.67) below).

\subsection{Scalar Field (analysed in terms of $L_{m}(x)$ for $m=2$ )}

As an example, consider the Lagrangian for the free scalar field $\phi(x, t)$, in units $\hbar=c=1$ :

$$
\mathcal{L}=\int_{0}^{N \ell}\left[\dot{\phi}^{\dagger} \dot{\phi}-\frac{\partial \phi^{\dagger}}{\partial x} \frac{\partial \phi}{\partial x}-\mu^{2} \phi^{\dagger} \phi\right] d x
$$


where $\mu$ is the mass, ${ }^{\dagger}$ denotes the Hermitian conjugation and the dot indicates the time derivative. The restriction to the zeroth band $n=0$ by using the lump function of order $m=2$ is identical to the expansion

$$
\phi(x, t)=\sum_{j} \phi_{j}(t) \Delta(x-j \ell)
$$

where $j$ goes over the $N$ lattice sites and $\Delta(x)$ is the triangular function given by (2.4). At the $j^{\text {th }}$ site $x=j \ell, \phi=\phi_{j}$ and the expansion (2.21) gives a simple linear interpolation, converting the set of discrete values $\left\{\phi_{j}\right\}$ to a continuous function. (The use of piecewise flat but continuous functions such as $\Delta(x-j \ell)$ for lattice formulation is the basis of discrete mechanics. ${ }^{4}$ )

We may substitute $(2.21)$ into $\mathcal{L}$ and derive the corresponding lattice equation by setting to zero the variational derivative of the action $\int \mathcal{L} d t$ with respect to $\phi_{j}(t)$. The resulting solution is

$$
\phi_{j}(t)=\sum_{K}\left[\frac{3}{N \ell(2+\cos \theta)}\right]^{\frac{1}{2}} e^{i j \theta} Q_{K}^{0}(t)
$$

where $Q_{K}^{0}(t)$ is the normal mode satisfying

$$
\ddot{Q}_{K}^{0}=-\left(w^{2}(\theta)+\mu^{2}\right) Q_{K}^{0}
$$

with

$$
w^{2}(\theta)=\frac{6(1-\cos \theta)}{\ell^{2}(2+\cos \theta)}
$$

and $\theta$ given by (2.8)-(2.9). Correspondingly, the lattice approximation (2.21) becomes

$$
\phi(x, t)=\sum_{K} Q_{K}^{0}(t) f_{0}(K \mid x)
$$

where the normalized $f_{0}(K \mid x)$ is

$$
f_{0}(K \mid x)=\left[\frac{3}{N \ell(2+\cos \theta)}\right]^{\frac{1}{2}} \sum_{j} e^{i j \theta} \Delta(x-j \ell) .
$$

Clearly, the full expansion in terms of all bands

$$
\phi(x, t)=\sum_{n} \sum_{K} Q_{K}^{n}(t) f_{n}(K \mid x)
$$


yields the exact continuum result. The details are given in I.

\section{Remarks.}

1. For $K$ within the Brillouin zone (i.e., $|\theta| \leq \pi$ ), the above frequency $\omega(K)=\sqrt{w^{2}(\theta)+\mu^{2}}$ is always higher than the exact continuum result $E(K)=\sqrt{K^{2}+\mu^{2}}$. This is expected since the expansion (2.21) may be regarded as a constraint imposed on the continuum problem; the corresponding optimal spectrum can only serve as an upper bound.

2. Instead of $\Delta(x-j \ell)$, in the expansion $(2.21)$ for $\phi(x, t)$ we may use another lump function $L_{m}(x-j \ell)$, but with $m>2$, as a constraint on the continuum field. (See, e.g., (2.28) below.) However, this does not work if $m$ is $<2$ : When $m=1, L_{1}(x)=\mathcal{C}(x)$ and its derivative is $\delta(x)-\delta(x-\ell)$, in accordance with (2.4); therefore, the application of the corresponding $0^{t h}$-band Bloch function to the continuum scalar field Lagrangian $\mathcal{L}$ would lead to $\infty$. (However, the $m=1$ lump function can be used as a link function for the gauge field, as will be discussed in Section 3.) When $m=0, L_{0}(x)=\delta(x / \ell)$; the substitution of the corresponding expansion of $\phi(x)$ into the continuum Lagrangian $\mathcal{L}$ would clearly also give $\infty$.

3. In this context, it may be useful to examine the usual discrete lattice of $N$ separate points. The corresponding Lagrangian $\mathcal{L}_{d}$ for a discrete field $\psi_{j}$ would be, instead of (2.20),

$$
\mathcal{L}_{d}=\sum_{j=1}^{N}\left[\dot{\psi}_{j}^{\dagger} \dot{\psi}_{j}-\left(\psi_{j+1}^{\dagger}-\psi_{j}^{\dagger}\right)\left(\psi_{j+1}-\psi_{j}\right) \ell^{-2}-\mu^{2} \psi_{j}^{\dagger} \psi_{j}\right]
$$

In terms of its normal modes we have, in place of (2.22)-(2.24),

$$
\begin{gathered}
\psi_{j}(t)=\sum_{K} N^{-\frac{1}{2}} e^{i j \theta} q_{K}(t), \\
\ddot{q}_{K}=-\omega_{d}(K)^{2} q_{K}
\end{gathered}
$$

with

$$
\omega_{d}(K)=\left[2(1-\cos \theta) \ell^{-2}+\mu^{2}\right]^{\frac{1}{2}}
$$

Note that the frequency $\omega_{d}(K)$ is lower than the continuum result $E(K)$, which underscores the difference (for $\ell \neq 0$ ) between a discrete lattice Lagrangian $\mathcal{L}_{d}$ 
and its continuum analog $\mathcal{L}$. When $\ell \rightarrow 0, \omega(K)$ and $\omega_{d}(K)$ both approach $E(K)$ with the deviations, $\omega(K)^{2}-E(K)^{2}$ and $\omega_{d}(K)^{2}-E(K)^{2}, O\left(\theta^{4}\right)$.

However, when $\ell \neq 0$, the usual discrete lattice Lagrangian $\mathcal{L}_{d}$ is more an abstract model of the continuum theory, rather than a systematic approximation of it. This is different from the direction that we follow, and the difference becomes especially prominent when we come to QCD (Sections 4 and 5). In Appendix A, we give a detailed comparison between these two approaches, which may help to explain the underlying reasons for constructing these special lump functions and for the particular way that we formulate our lattice approximation.

\subsection{Link Functions}

For $m$ odd, $L_{m}(x-j \ell)$ peaks at $x=\left(j+\frac{1}{2}\right) \ell$, which is the mid-point of the link connecting the $j^{t h}$ and $(j+1)^{t h}$ sites, located at $x=j \ell$ and $(j+1) \ell$. It is convenient to associate these functions with links, as will be discussed in later sections; therefore, the lump functions of odd order will also be called "link functions". Here, in one space-dimension, this difference is not an essential one since the mid-point of any link is also the lattice site in the dual structure. We shall illustrate the use of these link-functions by considering the special case $m=3$.

For the scalar field $\phi(x, t)$, instead of $(2.21)$, write

$$
\phi(x, t)=\sum \chi_{j}(t) S(x-j \ell)
$$

where $S(x)=L_{3}(x)$ is given by (2.4). Substituting this form into the Lagrangian (2.20) and varying $\chi_{j}(t)$, we find, similar to (2.22)-(2.26),

$$
\chi_{j}(t)=\sum_{K}\left[\frac{30}{N \ell\left(16+13 \cos \theta+\cos ^{2} \theta\right)}\right]^{\frac{1}{2}} e^{i\left(j+\frac{1}{2}\right) \theta} Q_{K}^{0}(t)
$$

where $Q_{K}^{0}(t)$ is the new normal mode satisfying

$$
\ddot{Q}_{K}^{0}=-\left(v^{2}(\theta)+\mu^{2}\right) Q_{K}^{0}
$$

with

$$
v^{2}(\theta)=\frac{20(1-\cos \theta)(2+\cos \theta)}{\ell^{2}\left(16+13 \cos \theta+\cos ^{2} \theta\right)}
$$


and $\theta$ given by (2.8)-(2.9), as before. Correspondingly, (2.28) becomes

$$
\phi(x, t)=\sum_{K} Q_{K}^{0}(t) g_{0}(K \mid x)
$$

where

$$
g_{0}(K \mid x)=\left[\frac{30}{N \ell\left(16+13 \cos \theta+\cos ^{2} \theta\right)}\right]^{\frac{1}{2}} \sum_{j} e^{i\left(j+\frac{1}{2}\right) \theta} S(x-j \ell) .
$$

For notational clarity, we denote the normalized zeroth-band Bloch function for the link functions by $g_{0}(K \mid x)$, instead of $f_{0}(K \mid x)$.

From (2.4), we see that $S\left(\frac{\ell}{2}\right)=\frac{3}{4}$ and $S\left(-\frac{\ell}{2}\right)=S\left(\frac{3 \ell}{2}\right)=\frac{1}{8}$. Thus, the value $\bar{\phi}_{j}(t) \equiv \phi(x, t)$ at the mid-point of each link (i.e., at $x=\left(j+\frac{1}{2}\right) \ell$ ) is related to $\chi_{j}(t)$ introduced in $(2.28)$ by

$$
\bar{\phi}_{j}=\frac{1}{8}\left(6 \chi_{j}+\chi_{j-1}+\chi_{j+1}\right) .
$$

The inverse relation is

$$
\chi_{j}=\sum_{\alpha} \sum_{j^{\prime}=1}^{N} e^{i\left(j-j^{\prime}\right) \alpha} 4[N(3+\cos \alpha)]^{-1} \bar{\phi}_{j^{\prime}}
$$

where

$$
\alpha=2 \pi n / N
$$

and its sum extends over $n=1,2, \cdot \cdot, N$. Both $\chi_{j}$ and $\bar{\phi}_{j}$ satisfy the periodic boundary condition $\chi_{j+N}=\chi_{j}$ and $\bar{\phi}_{j+N}=\bar{\phi}_{j}$.

In the usual lattice calculation, one tends to use $\bar{\phi}_{j}$ as the primary variables. To see how closely correlated the corresponding $\chi_{j}$ are, let us consider the special case $N \rightarrow \infty$ and

$$
\bar{\phi}_{j}= \begin{cases}1 & \text { for } j=0 \\ 0 & \text { otherwise }\end{cases}
$$

The corresponding $\chi_{j}$ is

$$
\chi_{j}=\sqrt{2} Z^{|j|} \cong(-)^{j} \sqrt{2} e^{-1.763|j|}
$$

where $Z=-3+2 \sqrt{2}$. Thus, $\chi_{j}$ decreases rapidly with increasing $|j|$. 
By following the steps $(2.16)-(2.18)$, we can start with the $0^{\text {th }}$ band linkfunction $g_{0}(K \mid x)$ and construct a complete set of orthonormal Bloch functions $\left\{g_{n}(K \mid x)\right\}$, satisfying

$$
\int_{0}^{N \ell} g_{n}(K \mid x)^{*} g_{n^{\prime}}\left(K^{\prime} \mid x\right) d x=\delta_{K K^{\prime}} \delta_{n n^{\prime}}
$$

The expansion of $\phi(x, t)$ in terms of all bands would, of course, give the correct continuum solution.

For $|\theta| \leq \pi$, like $w^{2}(\theta), v^{2}(\theta)$ is larger than the exact continuum eigenvalue $K^{2}=(\theta / \ell)^{2}$. This is again expected since the expansion (2.28) may also be regarded as a constraint imposed on the continuum problem. In addition, we have

$$
\delta(\theta) \equiv w^{2}(\theta)-v^{2}(\theta)=\frac{2(1-\cos \theta)^{2}(8+7 \cos \theta)}{\ell^{2}(2+\cos \theta)\left(16+13 \cos \theta+\cos ^{2} \theta\right)}>0,
$$

a relation that will be of use later on.

\subsection{Generalized Lump Functions}

The generalized lump functions are defined by

$$
L_{m, n}(x) \equiv \int_{-\infty}^{\infty} \frac{d k}{2 \pi} \frac{\left(2 \sin \frac{k}{2}\right)^{m}\left(\cos \frac{k}{2}\right)^{n}}{k^{m}} \times \begin{cases}e^{i k x / \ell} & \text { for } m+n \text { even } \\ e^{i k\left(x-\frac{1}{\ell}\right) / \ell} & \text { for } m+n \text { odd }\end{cases}
$$

where $m$ and $n$ are both positive integers. When $n=0, L_{m, n}(x)$ reduces to $L_{m}(x)$ given by $(2.1)$. These generalized functions are nonzero only within $m+n$ lattice cells. Similar to (2.2)-(2.3) and (2.5)-(2.6), we have for $m+n$ even

$$
\begin{gathered}
\ell \frac{d}{d x} L_{m, n}(x)=-L_{m-1, n}(x)+L_{m-1, n}(x+\ell), \\
\sum_{j=-(m+n-2) / 2}^{(m+n) / 2} L_{m, n}(x-j \ell)=\text { constant }
\end{gathered}
$$

and for $m+n$ odd 


$$
\begin{gathered}
\ell \frac{d}{d x} L_{m, n}(x)=L_{m-1, n}(x)-L_{m-1, n}(x-\ell), \\
\sum_{j=-(m+n-1) / 2}^{(m+n-1) / 2} L_{m, n}(x-j \ell)=\text { constant } .
\end{gathered}
$$

The following example for $m+n=4$ illustrates the variety of ways that we may interpolate from a discrete set of lattice values $\left\{\phi_{j}\right\}$ to a continuous function $\phi(x)$ in the continuum. Introduce a lump function of mixed orders:

$$
L(x) \equiv 3 L_{4,0}(x)-2 L_{3,1}(x)
$$

We find,

$$
L(x)= \begin{cases}\left(1-\frac{|x|}{\ell}\right)\left(1+\frac{|x|}{\ell}-\frac{3}{2} \frac{|x|^{2}}{\ell^{2}}\right) & \text { for }|x|<\ell \\ \frac{1}{2}\left(1-\frac{|x|}{\ell}\right)\left(2-\frac{|x|}{\ell}\right)^{2} & \text { for } \ell<|x|<2 \ell \\ 0 & \text { for }|x|>2 \ell\end{cases}
$$

Because $L(0)=1$ and $L( \pm \ell)=0$, the expansion

$$
\phi(x)=\sum_{j} \phi_{j} L(x-j \ell)
$$

gives $\phi\left(x_{j}\right)=\phi_{j}$ at all lattice sites $x_{j}=j \ell$, even though each $\phi_{j}$ influences the value of $\phi(x)$ over four nearby lattice cells (instead of two, as in the expansion (2.21) using $\Delta(x))$. The above lump function $L(x)$ is not always positive; it is 1 at $x=0$, decreases to 0 at $x= \pm \ell$, reaches a minimum $-2 / 27$ at $x= \pm \frac{4}{3} \ell$, then increases to 0 at $x= \pm 2 \ell$ and remains 0 for $|x|>2 \ell$. The corresponding normalized $0^{\text {th }}$ band Bloch function is, for $0<x<\ell$,

$$
\begin{aligned}
f_{0}(K \mid x)= & {\left[\frac{70}{N \ell\left(62+17 \cos \theta-10 \cos ^{2} \theta+\cos ^{3} \theta\right.}\right]^{\frac{1}{2}} } \\
& \times\left[e^{-i \theta} L(x+\ell)+L(x)+e^{i \theta} L(x-\ell)+e^{2 i \theta} L(x-2 \ell)\right]
\end{aligned}
$$

with $\theta$ given by (2.8)-(2.9). From (2.47), one can construct a complete set of orthonormal Bloch functions $\left\{f_{n}(K \mid x)\right\}$, as before. 


\section{QED (Free Field)}

The Lagrangian density of a free electromagnetic field $V_{\mu}(\vec{r}, t)$ is

$$
\mathcal{L}=-\frac{1}{4} F_{\mu \nu} F_{\mu \nu}
$$

where $F_{\mu \nu}=\frac{\partial V_{\nu}}{\partial x_{\mu}}-\frac{\partial V_{\mu}}{\partial x_{\nu}}$, and the repeated subscripts $\mu$ and $\nu$ are summed over $1,2,3$ and 4 (i.e., $x, y, z$ and it). We follow the standard procedure of deriving the continuum quantum Hamiltonian by first fixing the gauge. The next step is to choose an appropriate complete set of orthonormal $c$ number Bloch functions, in terms of which the quantized field operators can be expanded. The restriction to the $0^{\text {th }}$ band Bloch functions constitutes the lattice theory in the present approach, as in (2.27) and (2.25). A critical point for the gauge theory lies in being able to preserve the continuum gauge-fixing condition (valid at all continuous space-time coordinates) within the lattice approximation. Because of (1.2), the lattice gauge theory maintains the local character of the original field theory. The compatibility between the continuum gauge-fixing and the band decomposition makes it possible to carry out the transition from lattice to continuum in a straightforward and systematic way. As we shall discuss, the result is a new lattice gauge formulation, which retains the noncompact nature of the continuum gauge theory, and thereby may serve as its first approximation.

\subsection{Time-Axial Gauge}

In the time-axial gauge,

$$
V_{4}=i V_{0}=0 .
$$

Embed a cubic lattice of $\mathcal{N}=N^{3}$ sites in the continuum. Consider first the unit cubic cell (of volume $\ell^{3}$ ) which has the origin $(0,0,0)$ and the lattice-site $(1,1,1) \ell$ as two of its eight vertices. Altogether the cell borders on 12 links. Assign the values of $V_{x}$ on the four $x$ links (all parallel to the $x$-axis) to be the constants $a, a^{\prime}, a^{\prime \prime}$ and $a^{\prime \prime \prime}$. Likewise, on each of the $y$ and $z$ links $V_{y}$ and $V_{z}$ assume constant values $b, \cdot \cdot, b^{\prime \prime \prime}$ and $c, \cdot \cdot, c^{\prime \prime \prime}$, as shown in Figure 1. Inside the cell, let $V_{x}$ be a multilinear function of $y$ and $z$ :

$$
V_{x}=a+\left(a^{\prime}-a\right) \frac{y}{\ell}+\left(a^{\prime \prime}-a\right) \frac{z}{\ell}+\left(a^{\prime \prime \prime}-a^{\prime \prime}-a^{\prime}+a\right) \frac{y z}{\ell^{2}} ;
$$


similarly,

$$
V_{y}=b+\left(b^{\prime}-b\right) \frac{z}{\ell}+\left(b^{\prime \prime}-b\right) \frac{x}{\ell}+\left(b^{\prime \prime \prime}-b^{\prime \prime}-b^{\prime}+b\right) \frac{z x}{\ell^{2}}
$$

and

$$
V_{z}=c+\left(c^{\prime}-c\right) \frac{x}{\ell}+\left(c^{\prime \prime}-c\right) \frac{y}{\ell}+\left(c^{\prime \prime \prime}-c^{\prime \prime}-c^{\prime}+c\right) \frac{x y}{\ell^{2}} .
$$

The magnetic field $\vec{B}=\vec{\nabla} \times \vec{V}$ inside the cell is given by

$$
B_{x}=\ell^{-1}\left[\left(1-\frac{x}{\ell}\right)\left(c^{\prime \prime}-c-b^{\prime}+b\right)+\frac{x}{\ell}\left(c^{\prime \prime \prime}-c^{\prime}-b^{\prime \prime \prime}+b^{\prime \prime}\right)\right]
$$

and similar expressions for $B_{y}$ and $B_{z}$. Thus, on the $(y, z)$ plaquette at $x=0, B_{x}$ is a constant. From Figure 1, we see that this constant value $\ell^{-1}\left(c^{\prime \prime}-b^{\prime}-c+b\right)$ is equal to $\ell^{-1}$ times the algebraic sum of link-values along the counter-clockwise direction, with the signs determined by the arrow directions shown in the figure. Likewise, on the $(y, z)$ plaquette at $x=\ell, B_{x}$ is again a constant, $\ell^{-1}\left(c^{\prime \prime \prime}-b^{\prime \prime \prime}-c^{\prime}+b^{\prime \prime}\right)$. Inside the cell, $B_{x}$ depends linearly on $x$. Identical considerations can be applied to $B_{y}$ and $B_{z}$.

Next, we consider other cubic cells, each of which (say, the $j^{\text {th }}$ cell) can be arrived at through a space-translation, moving the corner of the above unit cell from the origin $(0,0,0)$ to the $j^{t h}$ lattice site at $\vec{r}_{j}=\left(j_{1}, j_{2}, j_{3}\right) \ell$. At the same time, replace the link-values $a, b$ and $c$ by $a_{j}, b_{j}$ and $c_{j}$. The entire $V_{\mu}$-function in the continuum space can then be obtained by following the same multilinear interpolation procedure (3.3)-(3.5). This will lead to the $0^{t h}$ band approximation in the new lattice gauge formulation. In this way, the $a_{j}, b_{j}$ and $c_{j}$ are defined once and for all on each link, so that in passing from one cell to another, there is no discontinuity of either the tangential components of $\vec{V}$ or the the normal components of $\vec{B}$. (See (3.6) above.)

As we shall see, by using the lump and link functions introduced in the previous section, we can cast the result in terms of Bloch functions consisting of products of (2.7). We write

$$
V_{a}=\sum_{\vec{K}} q_{a}(\vec{K}) g\left(K_{a} \mid x_{a}\right) \prod_{b \neq a} f\left(K_{b} \mid x_{b}\right)
$$

where the subscript $a$ or $b$ denotes the space-components 1,2 and 3 (or $x, y$ and $z), \vec{K}$ denotes the Bloch wave number, which is to be summed over the Brillouin zone with its components $K_{a}$ satisfying (2.8), (2.9) and (2.11); i.e. 


$$
K_{a} N \ell=2 \pi \times \text { integer }
$$

and the components of $\vec{\theta} \equiv \vec{K} \ell$ bounded by

$$
-\pi \leq \theta_{a} \leq \pi
$$

The functions $g\left(K_{a} \mid x_{a}\right)$ and $f\left(K_{b} \mid x_{b}\right)$ are

$$
g\left(K_{a} \mid x_{a}\right)=\left(\frac{1}{N \ell}\right)^{\frac{1}{2}} e^{\frac{i}{2} \theta_{a}} \sum_{n=-\infty}^{\infty} e^{i n \theta_{a}} C\left(x_{a}-n \ell\right)
$$

and

$$
f\left(K_{b} \mid x_{b}\right)=\left(\frac{3}{N \ell\left(2+\cos \theta_{b}\right)}\right)^{\frac{1}{2}} \sum_{n=-\infty}^{\infty} e^{i n \theta_{b}} \Delta\left(x_{b}-n \ell\right)
$$

where $C(x)$ and $\Delta(x)$ are the lump functions $L_{1}(x)$ and $L_{2}(x)$ introduced in the previous section. By referring to (2.4), we see that (3.7) reproduces (3.3)(3.5).

Consider again the $j^{\text {th }}$ cubic cell. By using (2.4), (3.7) and (3.10)-(3.11) we can write down immediately the values of $V_{x}, V_{y}$ and $V_{z}$ on the three links connected to the lattice site at $\vec{r}_{j}$. Thus,

$$
\begin{gathered}
a_{j}=\sum_{\vec{K}} \frac{1}{\sqrt{\Omega}} e^{i \vec{K} \cdot \vec{r}_{j}} e^{i \frac{1}{2} \theta_{1}}\left(\frac{3}{2+\cos \theta_{2}}\right)^{\frac{1}{2}}\left(\frac{3}{2+\cos \theta_{3}}\right)^{\frac{1}{2}} q_{1}(\vec{K}), \\
b_{j}=\sum_{\vec{K}} \frac{1}{\sqrt{\Omega}} e^{i \vec{K} \cdot \vec{r}_{j}} e^{i \frac{1}{2} \theta_{2}}\left(\frac{3}{2+\cos \theta_{3}}\right)^{\frac{1}{2}}\left(\frac{3}{2+\cos \theta_{1}}\right)^{\frac{1}{2}} q_{2}(\vec{K}), \\
c_{j}=\sum_{\vec{K}} \frac{1}{\sqrt{\Omega}} e^{i \vec{K} \cdot \vec{r}_{j}} e^{i \frac{1}{2} \theta_{3}}\left(\frac{3}{2+\cos \theta_{1}}\right)^{\frac{1}{2}}\left(\frac{3}{2+\cos \theta_{2}}\right)^{\frac{1}{2}} q_{3}(\vec{K})
\end{gathered}
$$

where $\Omega=(N \ell)^{3}$ is the total volume. (In this connection, it may be helpful to compare the conventional discretized Abelian lattice gauge theory to the present formulation based on using Boch wave functions, of which the zerothband ones are simple continuum interpolations of discrete values $a_{j}, b_{j}$ and $c_{j}$. A discussion is given in Appendix A.)

Substituting (3.3) and (3.7) into (3.1), we find the Lagrangian (in the $0^{\text {th }}$ band approximation) to be 


$$
\int \mathcal{L} d^{3} r=\sum_{\vec{K}}\left[\dot{q}^{\dagger}(\vec{K}) \dot{q}(\vec{K})-q^{\dagger}(\vec{K})\left(w^{2}-w \tilde{w}\right) q(\vec{K})\right]
$$

where $q(\vec{K})$ and $w=w(\vec{\theta})$ are $3 \times 1$ column matrices whose components are $q_{a}(\vec{K})$ and

$$
w_{a}(\vec{\theta})=\frac{2}{\ell} \sin \frac{\theta_{a}}{2}\left(\frac{3}{2+\cos \theta_{a}}\right)^{\frac{1}{2}}
$$

with $\vec{\theta}=\vec{K} \ell$ as before. Sometimes these column matrices will also be represented by vectors $\vec{q}(\vec{K})$ and $\vec{w}(\vec{\theta})$. In (3.13), denotes the transpose and

$$
w^{2}=\sum_{a=1}^{3} w_{a}(\vec{\theta})^{2}=\sum_{a=1}^{3} \frac{6\left(1-\cos \theta_{a}\right)}{\ell^{2}\left(2+\cos \theta_{a}\right)},
$$

which is the three-dimensional generalization of (2.24). Throughout the paper, $\sum_{\vec{K}}^{\prime}$ denotes the sum over half of the $\vec{K}$-space within the Brillouin zone. The corresponding Hamiltonian is

$$
H=\sum_{\vec{K}}\left[p^{\dagger}(\vec{K}) p(\vec{K})+q^{\dagger}(\vec{K})\left(w^{2}-w \tilde{w}\right) q(\vec{K})\right]
$$

with $p(\vec{K})=\dot{q}(\vec{K})$ satisfying the equal-time commutation relation

$$
\left[p_{a}(\vec{K}), q_{a^{\prime}}\left(\vec{K}^{\prime}\right)\right]=\delta_{\vec{K} \vec{K}^{\prime}} \delta_{a a^{\prime}}
$$

Both the Lagrangian and the Hamiltonian are invariant under the timeindependent gauge transformation

$$
\begin{gathered}
a_{j} \rightarrow a_{j}+\phi_{j^{\prime}}-\phi_{j}, \\
b_{j} \rightarrow b_{j}+\phi_{j^{\prime \prime}}-\phi_{j}, \\
c_{j} \rightarrow c_{j}+\phi_{j^{\prime \prime \prime}}-\phi_{j},
\end{gathered}
$$

where the subscripts refer to the sites

$$
\begin{aligned}
& j=\left(j_{1}, j_{2}, j_{3}\right), \quad j^{\prime}=\left(j_{1}+1, j_{2}, j_{3}\right), \\
& j^{\prime \prime}=\left(j_{1}, j_{2}+1, j_{3}\right) \text { and } j^{\prime \prime \prime}=\left(j_{1}, j_{2}, j_{3}+1\right) .
\end{aligned}
$$


This property can be readily established by noting that $\dot{\vec{V}}$ is unchanged since the transformation is independent of time and $\vec{B}=\vec{\nabla} \times \vec{V}$ is also not affected, on account of (3.6). As in the standard treatment in the time-axial gauge, the above symmetry necessitates the following constraint on the state vector $\mid>$ :

$$
\mathcal{J}_{j} \mid>=0
$$

where $\mathcal{J}_{j}$ is the generator associated with the transformation (3.17). There are altogether $\mathcal{N}-1$ such constraints. (Note that the special case $\phi_{1}=\phi_{2}=$ $\cdots=\phi_{\mathcal{N}}$ produces no change in $a_{j}, b_{j}$ and $c_{j}$; therefore (3.17) for all $j$ only imposes $\mathcal{N}-1$ constraints.)

In the Hilbert space representation in which the basis vectors are chosen to be the eigenstates $\mid q>$ of the operators $q_{a}(\vec{K})$, the state vector takes the form $<q \mid>$ and (3.19) becomes

$$
\mathcal{J}_{j}<q \mid>=0
$$

with $\mathcal{J}_{j}$ given by the differential operator

$$
\mathcal{J}_{j}=-i \sum_{\vec{K}}\left(\prod_{b=1}^{3} \sqrt{\frac{2+\cos \theta_{b}}{3 N}}\right) e^{-i \vec{K} \cdot \vec{r}_{j}} \sum_{a=1}^{3} w_{a} \frac{\partial}{\partial q_{a}(\vec{K})} .
$$

Hence, (3.20) is equivalent to, for $\vec{K} \neq 0$,

$$
\sum_{a=1}^{3} w_{a} \frac{\partial}{\partial q_{a}(\vec{K})}<q \mid>=0 .
$$

At a given $\vec{K}$, introduce a right-hand basis of three orthonormal vectors $\hat{e}_{1}(\vec{K}), \hat{e}_{2}(\vec{K})$ and $\hat{w}(\vec{K}) \equiv \vec{w} / \sqrt{w^{2}}$. Define the generalized coordinate for the longitudinal mode to be

$$
Q_{\ell}(\vec{K}) \equiv \hat{w}(\vec{K}) \cdot \vec{q}(\vec{K})
$$

and that for the transverse mode

$$
Q_{t}(\vec{K})=\hat{e}_{t}(\vec{K}) \cdot \vec{q}(\vec{K})
$$

where $t=1,2$ and $\vec{q}, \vec{w}$ are the vectorial forms of the column matrices $q$ and $w$. The constraint (3.22) implies $\langle q|>$ to be independent of all $Q_{\ell}(\vec{K})$. 
Thus, in the $0^{t h}$ band approximation, the spectrum of the free electromagnetic field is given by two transverse modes for each $\vec{K}$ in the Brillouin zone with $w^{2}(\vec{\theta})$ as the square of the energy. (Use of the subscript $\ell$ notation to denote "longitudinal" occurs only in this subsection and Appendix A.)

It is straightforward to construct higher band Bloch functions through (2.16)-(2.18). Expanding $V_{a}$ in terms of all bands, the spectrum changes from $w^{2}(\vec{\theta})$ to the exact continuum result $\vec{K}^{2}=\vec{\theta}^{2} / \ell^{2}$ for $\vec{K}$ within the Brillouin zone, as expected.

\section{Remarks:}

The inverse relation of (3.23)-(3.24),

$$
\vec{q}(\vec{K})=\hat{w}(\vec{K}) Q_{\ell}(\vec{K})+\sum_{t} \hat{e}_{t}(\vec{K}) Q_{t}(\vec{K}),
$$

enables us to decompose (3.7) into a sum of two parts:

$$
\vec{V}(\vec{r}, t)=\vec{V}_{\ell}(\vec{r}, t)+\vec{V}^{\prime}(\vec{r}, t)
$$

with their components given by

$$
\left(V_{\ell}\right)_{a}=\sum_{\vec{K}} \hat{w}(\vec{K})_{a} Q_{\ell}(\vec{K}) g\left(K_{a} \mid x_{a}\right) \prod_{b \neq a} f\left(K_{b} \mid x_{b}\right)
$$

and

$$
V_{a}^{\prime}=\sum_{\vec{K}} \sum_{t} \hat{e}_{t}(\vec{K})_{a} Q_{t}(\vec{K}) g\left(K_{a} \mid x_{a}\right) \prod_{b \neq a} f\left(K_{b} \mid x_{b}\right) .
$$

From (2.2) and (2.4), we have

$$
\ell \frac{d}{d x} \Delta(x)=-C(x)+C(x+\ell)
$$

which leads to the following relation between $g(K \mid x)$ and $f(K \mid x)$, defined by (3.10)-(3.11):

$$
\ell \frac{d}{d x_{a}} f\left(K_{a} \mid x_{a}\right)=i w_{a} g\left(K_{a} \mid x_{a}\right)
$$

with $w_{a}$ given by (3.14). Thus, $\vec{\nabla} \times \vec{V}_{\ell}=0$ at all points in the continuum space, since 


$$
\vec{V}_{\ell}(\vec{r}, t)=\vec{\nabla} \chi(\vec{r}, t)
$$

where

$$
\chi=\sum_{\vec{K}} \chi_{\vec{K}} \prod_{a} f\left(K_{a} \mid x_{a}\right)
$$

and

$$
\chi_{\vec{K}}=-i Q_{\ell}(\vec{K}) / \sqrt{w^{2}} .
$$

On the other hand, because $d g\left(K_{a} \mid x_{a}\right) / d x_{a}$ is very different from $f\left(K_{a} \mid x_{a}\right)$, one can readily verify

$$
\vec{\nabla} \cdot \vec{V}^{\prime} \neq 0 .
$$

Indeed, should we require $\vec{V}^{\prime}$ to be divergence free, the roles of $f$ and $g$ would have to be interchanged in (3.29), as will be discussed in the next section.

The state vector $\langle q|>$ introduced in (3.20) is a functional of $\vec{V}$ (i.e., of $\vec{V}_{\ell}$ and $\vec{V}^{\prime}$ ). The constraint (3.22) makes $\langle q|>$ to be a functional only of $\vec{V}^{\prime}(\vec{r}, t)$, independent of the longitudinal component $\vec{V}_{\ell}(\vec{r}, t)$. However, $\vec{\nabla} \cdot \vec{V}^{\prime} \neq$ 0 reminds us that the familiar difference between the time-axial gauge and the Coulomb gauge persists throughout the band structure.

We note that in the decomposition (3.25), the component $\hat{e}_{t}(\vec{K}) Q_{t}(\vec{K})$ is, of course, perpendicular (i.e., transverse) to the longitudinal component $\hat{w}(\vec{K}) Q_{\ell}(\vec{K})$. Because $\vec{K}$ lies within the Brillouin zone, this is only the lattice transversality condition; in contrast, (3.30) and (3.32) refer to relations in which $\vec{r}$ is a continuum coordinate.

\subsection{Coulomb Gauge}

Let $A_{\mu}(\vec{r}, t)$ be the electromagnetic field in the Coulomb gauge. Its spatial part $\vec{A}(\vec{r}, t)$ is divergence-free; i.e.,

$$
\vec{\nabla} \cdot \vec{A}=0 .
$$

The free-field Hamiltonian is

$$
H=\int \frac{1}{2}\left(\vec{\Pi}^{2}+\vec{B}^{2}\right) d^{3} r
$$


where $\vec{B}=\vec{\nabla} \times \vec{A}$ denotes the magnetic field and $-\vec{\Pi}$ is the electric field which also satisfies the transversality condition

$$
\vec{\nabla} \cdot \vec{\Pi}=0
$$

In addition, we have the equal-time commutation relation

$$
\left[\Pi_{a}(\vec{r}, t), A_{b}\left(\vec{r}^{\prime}, t\right)\right]=-i\left(\delta_{a b}-\nabla^{-2} \nabla_{a} \nabla_{b}\right) \delta^{3}\left(\vec{r}_{a}-\vec{r}_{b}\right)
$$

The central problem that confronts us is the construction of the appropriate $0^{\text {th }}$ band Bloch functions, so that (3.33) and (3.35) can be valid at all continuum points in the lattice approximation.

As mentioned at the end of the preceding section, in order to have a divergence-free field, the roles of $f$ and $g$ in (3.29) have to be interchanged. Thus, we replace the link function (3.10) by (2.33). We write, for the $0^{\text {th }}$ band function expansion,

$$
A_{a}(\vec{r}, t)=\sum_{\vec{K}} q_{a}(\vec{K}) F_{a}(\vec{K} \mid \vec{r})
$$

and

$$
\Pi_{a}(\vec{r}, t)=\sum_{\vec{K}} p_{a}(-\vec{K}) F_{a}(\vec{K} \mid \vec{r})
$$

where

$$
F_{a}(\vec{K} \mid \vec{r})=c_{3}\left(\theta_{a}\right) \mathcal{G}\left(K_{a} \mid x_{a}\right) \prod_{b \neq a} c_{2}\left(\theta_{b}\right) \mathcal{F}\left(K_{b} \mid x_{b}\right)
$$

in which, similar to (2.7), but with $m=2$ and 3 ,

$$
\mathcal{F}\left(K_{a} \mid x_{a}\right)=\sum_{n=-\infty}^{\infty} e^{i n \theta_{a}} \Delta\left(x_{a}-n \ell\right)
$$

and

$$
\mathcal{G}\left(K_{a} \mid x_{a}\right)=e^{i \frac{1}{2} \theta_{a}} \sum_{n=-\infty}^{\infty} e^{i n \theta_{a}} S\left(x_{a}-n \ell\right) .
$$

The factors

$$
c_{2}\left(\theta_{a}\right)=\left[\frac{3}{N \ell\left(2+\cos \theta_{a}\right)}\right]^{\frac{1}{2}}
$$


and

$$
c_{3}\left(\theta_{a}\right)=\left[\frac{30}{N \ell\left(16+13 \cos \theta_{a}+\cos ^{2} \theta_{a}\right)}\right]^{\frac{1}{2}},
$$

refer to the normalization constants $c_{m}$ in (2.15) with the subscripts $m=2$ and 3 denoting the order of the lump functions in $\mathcal{F}$ and $\mathcal{G}$. We note that apart from normalization factors, $\mathcal{F}$ and $\mathcal{G}$ are the same functions as $f$ of (3.11) and $g_{0}$ of $(2.33)$.

From (2.3)-(2.4), it follows that

$$
\ell \frac{d S(x)}{d x}=\Delta(x)-\Delta(x-\ell)
$$

and therefore

$$
\ell \frac{d}{d x_{a}} \mathcal{G}\left(K_{a} \mid x_{a}\right)=2 i \sin \frac{\theta_{a}}{2} \mathcal{F}\left(K_{a} \mid x_{a}\right)
$$

The transversality conditions (3.33) and (3.35) become simply

$$
\vec{q}(\vec{K}) \cdot \vec{v}(\vec{K})=0
$$

and

$$
\vec{p}(\vec{K}) \cdot \vec{v}(\vec{K})=0
$$

where the components of $\vec{v}(\vec{K})$ are

$$
v_{a}(\vec{K})=\frac{2}{\ell} \sin \frac{\theta_{a}}{2}\left[\frac{10\left(2+\cos \theta_{a}\right)}{16+13 \cos \theta_{a}+\cos ^{2} \theta_{a}}\right]^{\frac{1}{2}}
$$

Because

$$
F_{a}(\vec{K} \mid \vec{r})^{*}=F_{a}(-\vec{K} \mid \vec{r})
$$

we have

$$
q_{a}(\vec{K})=q_{a}(-\vec{K})^{\dagger} \quad \text { and } \quad p_{a}(\vec{K})=p_{a}(-\vec{K})^{\dagger}
$$

At a given $\vec{K}$, form a right-hand basis of three real orthonormal vectors $\hat{\epsilon}_{1}(\vec{K}), \hat{\epsilon}_{2}(\vec{K})$ and $\hat{v}(\vec{K}) \equiv \vec{v}(\vec{K}) / \sqrt{\vec{v}(\vec{K})^{2}}$ so that

$$
\hat{\epsilon}_{1}(\vec{K})=\hat{\epsilon}_{2}(\vec{K}) \times \hat{v}(\vec{K}) .
$$


Thus, if $\hat{\epsilon}_{2}(-\vec{K})=\hat{\epsilon}_{2}(\vec{K})$ then $\hat{\epsilon}_{1}(-\vec{K})=-\hat{\epsilon}_{1}(\vec{K})$, since $\hat{v}(-\vec{K})=-\hat{v}(\vec{K})$, in accordance with (3.44). Write

$$
q_{a}(\vec{K})=\sum_{t} \hat{\epsilon}_{t}(\vec{K})_{a} Q_{t}(\vec{K})
$$

and

$$
p_{a}(\vec{K})=\sum_{t} \hat{\epsilon}_{t}(\vec{K})_{a} P_{t}(\vec{K})
$$

where the sum is over $t=1,2$. The commutation relation (3.36), when applied to the $0^{\text {th }}$ band components of $\vec{\Pi}$ and $\vec{A}$, gives

$$
\left[P_{t}(\vec{K}), Q_{t^{\prime}}\left(\vec{K}^{\prime}\right)\right]=-i \delta_{\vec{K} \vec{K}^{\prime}} \delta_{t t^{\prime}},
$$

or in its equivalent form

$$
\left[p_{a}(\vec{K}), q_{a^{\prime}}\left(\vec{K}^{\prime}\right)\right]=-i \delta_{\vec{K} \vec{K}^{\prime}}\left(\delta_{a a^{\prime}}-\hat{v}_{a}(\vec{K}) \hat{v}_{a^{\prime}}(\vec{K})\right) .
$$

In the lattice approximation (3.37) and in terms of the column matrices

$$
p(\vec{K})=\left(\begin{array}{c}
p_{1}(\vec{K}) \\
p_{2}(\vec{K}) \\
p_{3}(\vec{K})
\end{array}\right) \quad \text { and } \quad q(\vec{K})=\left(\begin{array}{c}
q_{1}(\vec{K}) \\
q_{2}(\vec{K}) \\
q_{3}(\vec{K})
\end{array}\right)
$$

the Hamiltonian (3.34) becomes, on account of (3.28) and (3.43),

$$
H=\sum_{\vec{K}}\left[p^{\dagger} p+q^{\dagger} \Delta q\right]
$$

where the matrix $\Delta=\left(\Delta_{a b}(\vec{K})\right)$ is diagonal, with its diagonal elements

$$
\Delta_{a a}=w^{2}-\delta_{a},
$$

$w^{2}$ given by (3.15) and, similar to $(2.39), \delta_{a}=w_{a}^{2}-v_{a}^{2}$; i.e.,

$$
\delta_{a}=\frac{2\left(1-\cos \theta_{a}\right)^{2}\left(8+7 \cos \theta_{a}\right)}{\ell^{2}\left(2+\cos \theta_{a}\right)\left(16+13 \cos \theta_{a}+\cos ^{2} \theta_{a}\right)} .
$$

As before, $\theta_{a}=K_{a} \ell$. At a given $\vec{\theta}$, although $\Delta$ is a $3 \times 3$ matrix, because of the orthogonality condition (3.43), there are only two transverse modes whose 
eigen-frequencies $\omega^{2}=\omega_{ \pm}^{2}$ are determined by the secular equation (cf. Eq. (2.16) of I)

$$
\frac{v_{1}^{2}}{\Delta_{11}-\omega^{2}}+\frac{v_{2}^{2}}{\Delta_{22}-\omega^{2}}+\frac{v_{3}^{2}}{\Delta_{33}-\omega^{2}}=0 .
$$

The solution is

$$
\begin{aligned}
\omega_{ \pm}^{2}(\vec{\theta})= & w^{2}-\frac{1}{2 v^{2}}\left[v_{1}^{2}\left(\delta_{2}+\delta_{3}\right)+v_{2}^{2}\left(\delta_{3}+\delta_{1}\right)+v_{3}^{2}\left(\delta_{1}+\delta_{2}\right)\right] \\
& \pm \frac{1}{2 v^{2}}\left[\delta_{1}^{2}\left(v_{2}^{2}+v_{3}^{2}\right)^{2}+\delta_{2}^{2}\left(v_{3}^{2}+v_{1}^{2}\right)^{2}+\delta_{3}^{2}\left(v_{1}^{2}+v_{2}^{2}\right)^{2}\right. \\
& \left.\quad+2 \delta_{1} \delta_{2}\left(v_{1}^{2} v_{2}^{2}-v_{3}^{2} v^{2}\right)+2 \delta_{2} \delta_{3}\left(v_{2}^{2} v_{3}^{2}-v_{1}^{2} v^{2}\right)+2 \delta_{3} \delta_{1}\left(v_{3}^{2} v_{1}^{2}-v_{2}^{2} v^{2}\right)\right]^{\frac{1}{2}}
\end{aligned}
$$

where

$$
v^{2}=\sum_{a=1}^{3} v_{a}(\vec{\theta})^{2}=\sum_{a=1}^{3} \frac{20\left(1-\cos \theta_{a}\right)\left(2+\cos \theta_{a}\right)}{\ell^{2}\left(16+13 \cos \theta_{a}+\cos ^{2} \theta_{a}\right)} .
$$

Because $\delta_{a}>0$, each of the diagonal elements $\Delta_{a a}$ lies within $v^{2}$ and $w^{2}$. The two eigenvalues $\omega_{ \pm}^{2}$ have to be sandwiched between $\Delta_{11}, \Delta_{22}$ and $\Delta_{33}$; consequently, they must also satisfy

$$
v^{2}<\omega_{ \pm}^{2}<w^{2} .
$$

Using the spherical coordinates $v_{1}=v \sin \alpha \cos \beta, v_{2}=v \sin \alpha \sin \beta$ and $v_{3}=$ $v \cos \alpha$, we may express (3.56) in an alternative form:

$$
\omega_{ \pm}^{2}(\vec{\theta})=w^{2}+\frac{1}{2}\left[\lambda_{11}+\lambda_{22} \pm \sqrt{\left(\lambda_{11}-\lambda_{22}\right)^{2}+4 \lambda_{12}^{2}}\right]
$$

where

$$
\begin{gathered}
\lambda_{11}=-\delta_{1} \sin ^{2} \beta-\delta_{2} \cos ^{2} \beta, \\
\lambda_{22}=-\left(\delta_{1} \cos ^{2} \beta+\delta_{2} \sin ^{2} \beta\right) \cos ^{2} \alpha-\delta_{3} \sin ^{2} \alpha,
\end{gathered}
$$

and

$$
\lambda_{12}=\left(\delta_{1}-\delta_{2}\right) \sin \beta \cos \beta \cos \alpha .
$$

In the long wavelength region $\left|\theta_{a}\right|<<1, \omega_{ \pm}^{2}$ deviates from $w^{2}$ of (3.15) by $O\left(\theta^{4}\right)$, and (3.59) becomes

$$
\omega_{ \pm}^{2}=w^{2}-\frac{1}{24 \ell^{2}}\left[s_{2}-\frac{3 s_{3}}{s_{1}} \mp \sqrt{\left(s_{2}-\frac{s_{3}}{s_{1}}\right)\left(s_{2}-\frac{9 s_{3}}{s_{1}}\right)}\right]+O\left(\theta^{6}\right)
$$


where $s_{1}=\theta_{1}^{2}+\theta_{2}^{2}+\theta_{3}^{2}, s_{2}=\theta_{2}^{2} \theta_{3}^{2}+\theta_{3}^{2} \theta_{1}^{2}+\theta_{1}^{2} \theta_{2}^{2}$ and $s_{3}=\theta_{1}^{2} \theta_{2}^{2} \theta_{3}^{2}$.

The higher-band Bloch functions can be constructed through a generalization of (2.16)-(2.18). (See (3.73)-(3.79) below). As we shall see, these higherband Bloch functions are very close to the corresponding Fourier series of high wave numbers. By expanding $\vec{A}$ in terms of all bands, one changes the spectrum from the lattice approximation $\omega_{ \pm}^{2}$ to the continuum result.

\section{Remarks:}

1. By using (3.48), the $0^{\text {th }}$ band expansion (3.37) of $\vec{A}$ in the Coulomb gauge can be written as (with the time variable suppressed)

$$
\vec{A}(\vec{r})=\sum_{\vec{K}} \sum_{t=1}^{2} Q_{t}(\vec{K}) \vec{F}^{t}(\vec{K} \mid \vec{r})
$$

where $\vec{F}^{t}(\vec{K} \mid \vec{r})$ is a vector function whose components are

$$
F^{t}(\vec{K} \mid \vec{r})_{a}=\hat{\epsilon}_{t}(\vec{K})_{a} F_{a}(\vec{K} \mid \vec{r})
$$

Because of (3.38)-(3.42) and (3.47), for any $\vec{K}$ within the Brillouin zone

$$
\vec{\nabla} \cdot \vec{F}^{t}(\vec{K} \mid \vec{r})=0
$$

everywhere in the coordinate space. Thus, we have

$$
\vec{F}^{t}(\vec{K} \mid \vec{r})=\vec{\nabla} \times \vec{G}^{t}(\vec{K} \mid \vec{r})
$$

with the components of $\vec{G}^{t}(\vec{K} \mid \vec{r})$ given by

$$
G^{t}(\vec{K} \mid \vec{r})_{a}=i \sum_{t^{\prime}=1}^{2}\left(v^{2}\right)^{-\frac{1}{2}} \eta^{t t^{\prime}} \hat{\epsilon}_{t^{\prime}}(\vec{K})_{a} c_{2}\left(\theta_{a}\right) \mathcal{F}\left(K_{a} \mid x_{a}\right) \prod_{b \neq a} c_{3}\left(\theta_{b}\right) \mathcal{G}\left(K_{b} \mid x_{b}\right)
$$

and $\left(\eta^{t t^{\prime}}\right)$ denoting the $2 \times 2$ antisymmetric matrix whose elements are $\eta^{11}=$ $\eta^{22}=0$ and $\eta^{12}=-\eta^{21}=1$. Although the independent variables in the lattice approximation are restricted to the set $\left\{Q_{t}(\vec{K})\right\}$, the $0^{t h}$ band Bloch functions $\vec{F}^{t}(\vec{K} \mid \vec{r})$ satisfy the differential relations (3.63)-(3.64) at all $\vec{r}$ in the continuum. 
2. From (2.1) and (2.4), we see that the Fourier series expansion of (3.38) is

$$
F_{a}(\vec{K} \mid \vec{r})=e^{i \vec{K} \cdot \vec{r}} \sum_{\vec{m}} \mathcal{C}_{a}(\vec{\theta} \mid \vec{m}) e^{i 2 \pi \vec{m} \cdot \vec{r} / \ell}
$$

where

$$
\mathcal{C}_{a}(\vec{\theta} \mid \vec{m})=\left(\frac{2 \sin \frac{\theta_{a}}{2}}{\theta_{a}+2 \pi m_{a}}\right)^{3} c_{3}\left(\theta_{a}\right) \prod_{b \neq a}\left(\frac{2 \sin \frac{1}{2} \theta_{b}}{\theta_{b}+2 \pi m_{b}}\right)^{2} c_{2}\left(\theta_{b}\right),
$$

$m_{a}$ (the components of $\vec{m}$ ) are all integers, and $c_{2}, c_{3}$ are given by (3.40).

3. To construct the higher-band Bloch functions in the Coulomb gauge, we generalize the steps (2.12)-(2.16): Define

$$
\vec{\psi}_{\vec{p}}^{t}(\vec{r}) \equiv \Omega^{-\frac{1}{2}} \hat{e}^{t}(\vec{p}) e^{i \vec{p} \cdot \vec{r}}
$$

where $t=1,2$ denote two sets of divergence-free functions, with $\hat{e}^{1}$ and $\hat{e}^{2}$ both real unit vectors perpendicular to $\vec{p}$ and to each other. Resolve

$$
\vec{p}=\vec{K}+(2 \pi \vec{n} / \ell)
$$

with the components of $\vec{n}$ being any integer. At a given $\vec{K}$ within the Brillouin zone, write (3.68) as

$$
\vec{\psi}_{\vec{n}}^{t}(\vec{r})=\Omega^{-\frac{1}{2}} \hat{e}^{t}(\vec{n}) e^{i \vec{K} \cdot \vec{r}+i 2 \pi \vec{n} \cdot \vec{r} / \ell}
$$

Introduce the $2 \times 2$ matrix $T_{\vec{n}}$ whose elements are

$$
T_{\vec{n}}^{t t^{\prime}}(\vec{K}) \equiv \int \vec{F}^{t}(\vec{K} \mid \vec{r}) \cdot \vec{\psi}_{\vec{n}} t^{\prime}(\vec{r})^{*} d^{3} r
$$

where $\vec{F}^{t}(\vec{K} \mid \vec{r})$ is given by (3.62). The complete set of orthonormal divergencefree Bloch functions $\left\{\vec{F}_{\vec{n}}^{t}(\vec{K} \mid \vec{r})\right\}$ can be constructed by requiring, for $\vec{n}=0$,

$$
\vec{F}_{0}^{t}(\vec{K} \mid \vec{r})=\vec{F}^{t}(\vec{K} \mid \vec{r})
$$

and for $\vec{n} \neq 0$

$$
\vec{F}_{\vec{n}}^{t}(\vec{K} \mid \vec{r})=\vec{\psi}_{\vec{n}}^{t}(\vec{r})-\sum_{t^{\prime}=1}^{2}\left[\left(M_{\vec{n}}\right)^{t t^{\prime}} \vec{F}_{0}^{t^{\prime}}(\vec{K} \mid \vec{r})+\left(N_{\vec{n}}\right)^{t t^{\prime}} \vec{\psi}_{0} t^{\prime}(\vec{r})\right]
$$


The orthogonality between $\vec{F}_{0}^{t}$ and $\vec{F}_{\vec{n}}^{t^{\prime}}$ yields the $2 \times 2$ matrix relation

$$
T_{\vec{n}}-M_{\vec{n}}^{\dagger}-T_{0} N_{\vec{n}}^{\dagger}=0
$$

which together with the orthonormality between $\vec{F}_{\vec{n}}^{t}$ and $\vec{F}_{\vec{m}}^{t^{\prime}}$ for nonzero $\vec{n}$ and $\vec{m}$ give

$$
M_{\vec{n}} M_{\vec{m}}^{\dagger}=N_{\vec{n}} N_{\vec{m}}^{\dagger}
$$

The solution of $(3.75)$ is

$$
N_{\vec{n}}=M_{\vec{n}} u
$$

with $u$ an arbitrary $2 \times 2$ unitary matrix satisfying

$$
u u^{\dagger}=1
$$

By using (3.74), we have

$$
M_{\vec{n}}=T_{\vec{n}}^{\dagger}\left(1+u T_{0}^{\dagger}\right)^{-1}
$$

and

$$
N_{\vec{n}}=T_{\vec{n}}^{\dagger}\left(1+u T_{0}^{\dagger}\right)^{-1} u
$$

It follows then (for all $\vec{n}$ and $\vec{m}$, including 0 ) that the orthonormality relation

$$
\int_{\Omega} d^{3} r \vec{F}_{\vec{n}}^{t}(\vec{K} \mid \vec{r})^{*} \cdot \vec{F}_{\vec{m}}^{t^{\prime}}\left(\vec{K}^{\prime} \mid \vec{r}\right)=\delta_{\vec{n} \vec{m}} \delta_{t t^{\prime}} \delta_{\vec{K} \vec{K}^{\prime}}
$$

in addition, every member of the set $\left\{\vec{F}_{\vec{n}}^{t}\right\}$ satisfies the divergence-free condition

$$
\vec{\nabla} \cdot \vec{F}_{\vec{n}}^{t}(\vec{K} \mid \vec{r})=0
$$

If one wishes, the arbitrary $u$ can be set to 1 ; (3.78) becomes simply

$$
M_{\vec{n}}=N_{\vec{n}}=T_{\vec{n}}^{\dagger}\left(1+T_{0}^{\dagger}\right)^{-1}
$$

Because of (3.67), the matrix elements

$$
T_{\vec{n}}^{t t^{\prime}}=\sum_{a=1}^{3} \hat{\epsilon}^{t}(\vec{K})_{a} \cdot \hat{e}^{t^{\prime}}(\vec{n})_{a} \mathcal{C}_{a}(\vec{\theta} \mid \vec{n})
$$


are all real. The expansion of $\vec{A}(\vec{r}, t)$ and $\vec{\Pi}(\vec{r}, t)$ in terms of all bands,

$$
\vec{A}(\vec{r}, t)=\sum_{\vec{n}, \vec{K}, t} Q_{\vec{n}}^{t}(\vec{K}) \vec{F}_{\vec{n}}^{t}(\vec{K} \mid \vec{r})
$$

and

$$
\vec{\Pi}(\vec{r}, t)=\sum_{\vec{n}, \vec{K}, t} P_{\vec{n}}^{t}(-\vec{K}) \vec{F}_{\vec{n}}^{t}(\vec{K} \mid \vec{r})
$$

changes the $0^{\text {th }}$ band spectrum from the lattice approximation $\omega_{ \pm}^{2}(\vec{\theta})$ of $(3.56)$ to the exact continuum result $\vec{K}^{2}=(\vec{\theta} / \ell)^{2}$ within the Brillouin zone and, for $\vec{n} \neq 0,[\vec{K}+(2 \pi \vec{n} / \ell)]^{2}$ outside the zone.

4. The gauge-fixing conditions, $V_{0}(\vec{r}, t)=0$ in the time-axial gauge and $\vec{\nabla} \cdot \vec{A}(\vec{r}, t)=0$ in the Coulomb gauge, are valid at all $\vec{r}$ in the lattice approximation. This necessitates the difference between the $0^{\text {th }}$ band functions in these two gauges. Of course, in either gauge, the Bloch functions of all bands are complete. The transformation matrix between these different band structures can therefore be readily derived.

5. Similar considerations are also applicable to a generalized Coulomb-like gauge where, instead of $\vec{\nabla} \cdot \vec{A}=\nabla_{a} A_{a}=0$, we have ${ }^{5}$ at all continuum $\vec{r}$

$$
\int\left(\vec{r}\left|\Gamma_{a}\right| \vec{r}^{\prime}\right) A_{a}\left(\vec{r}^{\prime}\right) d^{3} r^{\prime}=0,
$$

with $\Gamma_{a}$ a linear operator independent of $\vec{A}$. Accordingly, we have to create a new class of lump functions of different orders $m$, for which relations like (2.2)-(2.3) remain valid, but with the differential operator $\partial / \partial x_{a}$ replaced by $\Gamma_{a}$. Details will be given elsewhere.

The extension to QCD in a Coulomb or Coulomb-like gauge is straightforward since the group index is external to the band decomposition, as will be discussed in the next section. However, as mentioned in the Introduction, the extension to QCD in the time-axial gauge is more complicated: While the gauge condition $A_{4}(\vec{r}, t)=0$ being a linear equation offers no problem in the band decomposition, the generalization of the constraint equation (3.19)

$$
\mathcal{J}_{j} \mid>=0
$$


becomes nonlinear in QCD. That means in solving this constraint, there would be additional coupling between different bands which must be taken into account.

In the next two sections, we will discuss QCD, but only in Coulomb gauge. 


\section{QUANTUM CHROMODYNAMICS}

Let $\mathcal{A}_{a}^{\ell}(\vec{r}, t)$ be the field in the Coulomb gauge, with the subscript $a=$ $1,2,3$ denoting its spatial components and the superscript $\ell=1,2, \cdot \cdot, n^{2}-$ 1 its $S U(n)$ index. The conjugate momentum is $\mathcal{P}_{a}^{\ell}(\vec{r}, t)$. Both satisfy the divergence-free condition

$$
\vec{\nabla} \cdot \overrightarrow{\mathcal{A}}^{\ell}(\vec{r}, t)=\vec{\nabla} \cdot \overrightarrow{\mathcal{P}}^{\ell}(\vec{r}, t)=0
$$

and the equal-time commutation relation

$$
\left[\mathcal{P}_{a}^{\ell}(\vec{r}, t), \mathcal{A}_{a^{\prime}}^{\ell^{\prime}}\left(\vec{r}^{\prime}, t\right)\right]=-i \delta^{\ell \ell^{\prime}}\left(\delta_{a b}-\nabla^{-2} \nabla_{a} \nabla_{b}\right) \delta^{3}\left(\vec{r}-\vec{r}^{\prime}\right)
$$

The continuum QCD Hamiltonian (without quarks) is ${ }^{5}$

$$
\mathcal{H}=\frac{1}{2} \int\left[\mathcal{J}^{-1} \mathcal{P}_{a}^{\ell} \mathcal{J} \mathcal{P}_{a}^{\ell}+\mathcal{B}_{a}^{\ell} \mathcal{B}_{a}^{\ell}\right] d^{3} r+\mathcal{H}_{\text {Coul }}
$$

where the Coulomb interaction is

$$
\begin{gathered}
\mathcal{H}_{\text {Coul }}=\frac{1}{2} g_{0}^{2} \mathcal{J}^{-1} \int \sigma^{\ell}(\vec{r})\left(\ell, \vec{r}\left|\left(\nabla_{a} \mathcal{D}_{a}\right)^{-1}\left(-\nabla^{2}\right)\left(\nabla_{b} \mathcal{D}_{b}\right)^{-1}\right| \ell^{\prime}, \vec{r}^{\prime}\right) \\
\cdot \mathcal{J} \sigma^{\ell^{\prime}}\left(\vec{r}^{\prime}\right) d^{3} r d^{3} r^{\prime}
\end{gathered}
$$

with the charge density

$$
\sigma^{\ell}=f^{\ell m n} \mathcal{A}_{a}^{m} \mathcal{P}_{a}^{n} ;
$$

the color magnetic field $\mathcal{B}_{a}^{\ell}$ is given by

$$
\epsilon_{a b c} \mathcal{B}_{c}^{\ell}=\nabla_{a} \mathcal{A}_{b}^{\ell}-\nabla_{b} \mathcal{A}_{a}^{\ell}+g_{0} f^{\ell m n} \mathcal{A}_{a}^{m} \mathcal{A}_{b}^{n}
$$

$\mathcal{D}_{a}$ denotes the covariant derivative matrix whose elements are

$$
\mathcal{D}_{a}^{\ell m}=\delta^{\ell m} \nabla_{a}-g_{0} f^{\ell m n} \mathcal{A}_{a}^{n},
$$

$\mathcal{J}$ is the the Jacobian ${ }^{6}$, or the Faddeev-Popov determinant ${ }^{7}$

$$
\mathcal{J}=\operatorname{det}\left|\nabla_{a} \mathcal{D}_{a}\right|
$$


$\epsilon_{a b c}$ is the usual antisymmetric tensor in the three-dimensional space, $f^{\ell m n}$ is the antisymmetric structure constant of the $S U(n)$ group algebra and $g_{0}$ is the bare coupling constant, related to the renormalized coupling $g$ by

$$
g_{0}=g+\delta g
$$

as will be discussed later. All the above repeated indices are summed over (but not for the equations below).

\section{1. $0^{\text {th }}$ Band Expansion}

The set $\vec{F}_{\vec{n}}^{t}(\vec{K} \mid \vec{r})$, given by (3.72)-(3.73), forms a complete orthonormal functional basis that satisfies $\left\{\vec{\nabla} \cdot \vec{F}_{\vec{n}}^{t}(\vec{K} \mid \vec{r})\right\}=0$. Consequently, we can always expand the field operators $\overrightarrow{\mathcal{A}}^{\ell}(\vec{r}, t)$ and $\overrightarrow{\mathcal{P}} \ell(\vec{r}, t)$ in terms of these Bloch functions, similar to (3.83). In the $0^{\text {th }}$ band approximation, we restrict the expansion only to $\vec{n}=0$. For clarity, the $0^{t h}$ band approximations of $\overrightarrow{\mathcal{A}}^{\ell}(\vec{r}, t)$ and $\overrightarrow{\mathcal{P}}^{\ell}(\vec{r}, t)$ will be denoted by $\vec{A}^{\ell}(\vec{r}, t)$ and $\vec{\Pi}^{\ell}(\vec{r}, t)$, whose components are given by, analogous to (3.37),

$$
A_{a}^{\ell}(\vec{r}, t)=\sum_{\vec{K}} q_{a}^{\ell}(\vec{K}) F_{a}(\vec{K} \mid \vec{r})
$$

and

$$
\Pi_{a}^{\ell}(\vec{r}, t)=\sum_{\vec{K}} p_{a}^{\ell}(-\vec{K}) F_{a}(\vec{K} \mid \vec{r})
$$

where $F_{a}(\vec{K} \mid \vec{r})$ is given by (3.38). As in (3.43) and (3.50), the divergence-free condition (4.1) requires

$$
\sum_{a=1}^{3} q_{a}^{\ell}(\vec{K}) v_{a}(\vec{K})=\sum_{a=1}^{3} p_{a}^{\ell}(\vec{K}) v_{a}(\vec{K})=0
$$

and the commutation relation (4.2) leads to

$$
\left[p_{a}^{\ell}(\vec{K}), q_{a^{\prime}}^{\ell^{\prime}}\left(\vec{K}^{\prime}\right)\right]=-i \delta^{\ell \ell^{\prime}} \delta_{\vec{K} \vec{K}^{\prime}}\left(\delta_{a a^{\prime}}-\hat{v}_{a}(\vec{K}) \hat{v}_{a^{\prime}}(\vec{K})\right)
$$

The substitution of (4.10)-(4.11) into (4.3)-(4.4) gives the noncompact lattice formulation of QCD; in addition, $g_{0}$ should be replaced by $g_{\ell}$, the lattice coupling constant, as will be discussed in Section 5. (See (5.1)-(5.6) below.) 
Because of (3.38)-(3.39), the Bloch function $F_{a}(\vec{K} \mid \vec{r})$ satisfies the lattice locality condition (1.2). To appreciate its consequences, we express (4.10), the expansion of $\vec{A}^{\ell}(\vec{r}, t)$, in an equivalent form:

$$
\begin{aligned}
& A_{x}^{\ell}(\vec{r}, t)=\sum_{j} a_{j}^{\ell}(t) S\left(x-j_{1} \ell\right) \Delta\left(y-j_{2} \ell\right) \Delta\left(z-j_{3} \ell\right), \\
& A_{y}^{\ell}(\vec{r}, t)=\sum_{j} b_{j}^{\ell}(t) \Delta\left(x-j_{1} \ell\right) S\left(y-j_{2} \ell\right) \Delta\left(z-j_{3} \ell\right)
\end{aligned}
$$

and

$$
A_{z}^{\ell}(\vec{r}, t)=\sum_{j} c_{j}^{\ell}(t) \Delta\left(x-j_{1} \ell\right) \Delta\left(y-j_{2} \ell\right) S\left(z-j_{3} \ell\right)
$$

where $\Delta(x)$ and $S(x)$ are the lump functions given by (2.4), and $j$ denotes the $j^{\text {th }}$ lattice site located at $\vec{r}_{j}=\left(j_{1}, j_{2}, j_{3}\right) \ell$. On account of $(3.41)$, the $\vec{\nabla} \cdot \vec{A}^{\ell}=0$ condition gives

$$
a_{j}^{\ell}-a_{j_{-}^{\prime}}^{\ell}+b_{j}^{\ell}-b_{j_{-}^{\prime \prime}}^{\ell}+c_{j}^{\ell}-c_{j_{-}^{\prime \prime \prime}}^{\ell}=0
$$

where the subscripts refer to the sites

$$
\begin{gathered}
j=\left(j_{1}, j_{2}, j_{3}\right), \quad j_{-}^{\prime}=\left(j_{1}-1, j_{2}, j_{3}\right), \\
j_{-}^{\prime \prime}=\left(j_{1}, j_{2}-1, j_{3}\right) \quad \text { and } \quad j_{-}^{\prime \prime \prime}=\left(j_{1}, j_{2}, j_{3}-1\right) .
\end{gathered}
$$

Equation (4.15) is the familiar field-flux conservation relation on a lattice (analogous to the Kirchhoff law). In our case, this discrete form co-exists with its continuum realization $\vec{\nabla} \cdot \vec{A}=0$ at all $\vec{r}$.

(A comment on notation: Whenever the letter $\ell$ appears as a superscript, it denotes the color index. Otherwise, it may designate the lattice spacing, as in $x-j_{1} \ell$ above, or it may indicate quantities associated with a lattice; e.g., the lattice coupling constant $g_{\ell}$ that will be introduced later.)

Figure 2 gives a simple example of a localized configuration with

$$
\begin{gathered}
A_{x}^{\ell}=S(x)[\Delta(y)-\Delta(y-\ell)] \Delta(z) \delta^{\ell 1}, \\
A_{y}^{\ell}=-[\Delta(x)-\Delta(x-\ell)] S(y) \Delta(z) \delta^{\ell 1},
\end{gathered}
$$

and

$$
A_{z}^{\ell}=0
$$


(the factor $\delta^{\ell 1}$ can be replaced by any other distribution in the $S U(n)$ index $\ell)$. As explained in the caption, by examining the circulation of link fluxes associated with the four links of the central plaquette in Figure 2, one sees that (4.15) is fulfilled. Through direct differentiation, this configuration also satisfies $\vec{\nabla} \cdot \vec{A}^{\ell}=0$ everywhere; it can be readily verifed that there are altogether $2 \times 9$ cubic cells with nonzero $\vec{A}^{\ell}$. The number inside each of the nine plaquettes (all located at $z=0$ ) in Figure 2 denotes the corresponding integral $\ell^{-1} \int B_{z}^{1} d x d y$ over the plaquette.

\subsection{Lattice Variables in the Coordinate Space}

In the Bloch wave-number space, the generalized coordinates $q_{a}^{\ell}(\vec{K})$ in the expansion (4.10) satisfy

$$
q_{a}^{\ell}(\vec{K})=q_{a}^{\ell}(-\vec{K})^{\dagger}
$$

and the transversality condition (4.12). At a given superscript $\ell$ and for each pair $\pm \vec{K} \neq 0$, we can construct four independent Hermitian variables from the two combinations

$$
Q_{t}^{\ell}(\vec{K}) \pm Q_{t}^{\ell}(\vec{K})^{\dagger}
$$

each with the index $t=1$ or 2 , where

$$
Q_{t}^{\ell}(\vec{K}) \equiv \sum_{a=1}^{3} \hat{\epsilon}_{t}(\vec{K})_{a} q_{a}^{\ell}(\vec{K})
$$

and $\hat{\epsilon}_{t}(\vec{K})$ given by (3.47). Thus, for a finite lattice of $\mathcal{N}=N^{3}$ unit cubic cells with periodic boundary conditions, there are $2(\mathcal{N}-1)$ independent Hermitian variables for $\vec{K} \neq 0$, and for $\vec{K}=0$

$$
3 \text { constant } A_{a}^{\ell} \text { solutions, }
$$

making a total of

$$
2 \mathcal{N}+1
$$

independent Hermitian variables in the Coulomb gauge, all explicitly exhibited. Here, as well as throughout this and the next subsections, we keep the group superscript $\ell$ fixed; therefore in the counting of degrees of freedom we do not include the trivial factor $n^{2}-1$ due to the $S U(n)$ generators. 
However, the same problem appears in a different form, if one wishes to use the coordinate space lattice variables. We note that the expansion (4.14) employs $3 \mathcal{N}$ Hermitian variables

$$
\left\{a_{j}^{\ell}, b_{j}^{\ell}, c_{j}^{\ell}\right\}
$$

Because of (4.15), there are $\mathcal{N}-1$ constraints (in which the subtraction of 1 is on account of the sum of (4.15) over all $j$ being 0 identically); this leads to the same total number $3 \mathcal{N}-(\mathcal{N}-1)=2 \mathcal{N}+1$ independent variables, given by (4.22).

Excluding the three constant $\vec{K}=0$ solutions (mentioned in (4.21)), we may write

$$
\vec{A}^{\ell}=\vec{\nabla} \times \vec{I}^{\ell}
$$

where the spatial components of $\vec{I}^{\ell}$ are given by

$$
\begin{aligned}
I_{x}^{\ell} & =\ell \sum_{j} \xi_{j}^{\ell} \Delta\left(x-j_{1} \ell\right) S\left(y-j_{2} \ell\right) S\left(z-j_{3} \ell\right), \\
I_{y}^{\ell} & =\ell \sum_{j} \eta_{j}^{\ell} S\left(x-j_{1} \ell\right) \Delta\left(y-j_{2} \ell\right) S\left(z-j_{3} \ell\right), \\
I_{z}^{\ell} & =\ell \sum_{j} \zeta_{j}^{\ell} S\left(x-j_{1} \ell\right) S\left(y-j_{2} \ell\right) \Delta\left(z-j_{3} \ell\right),
\end{aligned}
$$

with the factor $\ell$ denoting the lattice spacing, as before. Clearly, $\vec{A}^{\ell}$ and, therefore, also (4.23) are invariant under

$$
\vec{I}^{\ell} \rightarrow \vec{I}^{\ell}+\vec{\nabla} \chi^{\ell} .
$$

For a lattice interpretation of these continuum relations, we specify

$$
\chi^{\ell}=\ell^{2} \sum_{j} \chi_{j}^{\ell} S\left(x-j_{1} \ell\right) S\left(y-j_{2} \ell\right) S\left(z-j_{3} \ell\right) .
$$

Because of (3.41), (4.24) can be expressed in terms of the discrete variables:

$$
\begin{aligned}
& a_{j}^{\ell}=\zeta_{j}^{\ell}-\zeta_{j_{-}^{\prime \prime}}^{\ell}-\eta_{j}^{\ell}+\eta_{j_{-}^{\prime \prime}}^{\ell}, \\
& b_{j}^{\ell}=\xi_{j}^{\ell}-\xi_{j_{-}^{\prime \prime \prime}}^{\ell}-\zeta_{j}^{\ell}+\zeta_{j_{-}^{\prime}}^{\ell}, \\
& c_{j}^{\ell}=\eta_{j}^{\ell}-\eta_{j_{-}^{\prime}}^{\ell}-\xi_{j}^{\ell}+\xi_{j_{-}^{\prime \prime}}^{\ell}
\end{aligned}
$$


similarly, the transformation (4.26) takes the discrete form

$$
\begin{aligned}
& \xi_{j}^{\ell} \rightarrow \xi_{j}^{\ell}+\chi_{j}^{\ell}-\chi_{j_{-}^{\prime}}^{\ell}, \\
& \eta_{j}^{\ell} \rightarrow \eta_{j}^{\ell}+\chi_{j}^{\ell}-\chi_{j_{-}^{\prime \prime}}^{\ell}, \\
& \zeta_{j}^{\ell} \rightarrow \zeta_{j}^{\ell}+\chi_{j}^{\ell}-\chi_{j_{-}^{\prime \prime \prime}}^{\ell},
\end{aligned}
$$

where the lattice sites $j, j_{-}^{\prime}, j_{-}^{\prime \prime}$ and $j_{-}^{\prime \prime \prime}$ are given by (4.16).

As remarked before, we may identify $a_{j}^{\ell}, b_{j}^{\ell}$ and $c_{j}^{\ell}$ as "link-fluxes" which flow along the three links connecting the site $j$ to sites $j^{\prime}, j^{\prime \prime}$ and $j^{\prime \prime \prime}$, given by (3.18). Consider the cubic cell in which $x-j_{1} \ell, y-j_{2} \ell$ and $z-j_{3} \ell$ are all between 0 and $\ell$. Label its plaquette shared by links $\overline{j j}^{\prime \prime}$ and $\overline{j j}^{\prime \prime \prime}$ as $X_{j}$; likewise the plaquette $Y_{j}$ is shared by links $\overline{j j}^{\prime \prime \prime}$ and $\overline{j j}^{\prime}$, and the plaquette $Z_{j}$ by $\overline{j j}^{\prime}$ and $\overline{j j}^{\prime \prime}$, as shown in Figure $3(\mathrm{a})$. Identify $\xi_{j}^{\ell}, \eta_{j}^{\ell}$ and $\zeta_{j}^{\ell}$ as the circulating "plaquette-fluxes" which flow counterclockwise along the edges of plaquettes $X_{j}, Y_{j}$ and $Z_{j}$, as illustrated by Figure $3(\mathrm{~b})$. (For example, $\xi_{j}^{\ell}$ resembles the current loop represented by a magnetic moment pointing in the $x$ direction, that is normal to $X_{j}$. The equation $\vec{A}=\vec{\nabla} \times \vec{I}$ resembles $\vec{J}=\vec{\nabla} \times \vec{M}$ from electromagnetism.)

The continuum relation $\vec{A}^{\ell}=\vec{\nabla} \times \vec{I}^{\ell}$ implies $\vec{\nabla} \cdot \vec{A}^{\ell}=0$. The discrete expression (4.28) carries the same implication: Each link is shared by four plaquettes; through superposition of these plaquette-fluxes, the net flux along each link is given by (4.28), which automatically satisfies Kirchhoff's law (4.15). To summarize: in accordance with (4.21)-(4.22), there are $2 \mathcal{N}+1$ independent link-flux variables $\left\{a_{j}^{\ell}, b_{j}^{\ell}, c_{j}^{\ell}\right\}$; excluding the three constant $A_{a}^{\ell}$ solutions, the remaining $2 \mathcal{N}+1-3=2 \mathcal{N}-2$ independent link-flux variables can all be generated by the plaquette-fluxes through (4.28). In other words, among the $3 \mathcal{N}$ plaquette-flux variables $\left\{\xi_{j}^{\ell}, \eta_{j}^{\ell}, \zeta_{j}^{\ell}\right\}$, there are

$$
3 \mathcal{N}-(2 \mathcal{N}-2)=\mathcal{N}+2
$$

redundant ones that imply no net link-flux. (See Section 4.3 below.) The problem of eliminating the redundant variables can then be shifted from link-fluxes to plaquette-fluxes.

In the lattice formulation of QCD presented in this section, there is a precise connection relating the continuum equations (4.1), (4.24) and (4.26) with their 
discrete counterparts given by (4.15), (4.28) and (4.29). The discrete realizations can be further developed through the interplay between the lattice and its dual structure as follows:

In the dual lattice, let links $\bar{x}_{j}, \bar{y}_{j}, \bar{z}_{j}$ be duals to plaquettes $X_{j}, Y_{j}, Z_{j}$

in the original lattice. The plaquette-fluxes $\xi_{j}^{\ell}, \eta_{j}^{\ell}$, and $\zeta_{j}^{\ell}$ can also be regarded as "currents" in the dual lattice, all flowing along the respective links. In the same dual lattice, assign to each lattice site $j$ a "potential" $\chi_{j}^{\ell}$; transformation (4.29) generates a corresponding current change along each link by an amount equal to the potential difference between its two end points. Such a change does not alter the link-fluxes $\left(a_{j}^{\ell}, b_{j}^{\ell}, c_{j}^{\ell}\right)$ in the original lattice. These extra degrees of freedom enable us to eliminate $\mathcal{N}-1$ of the $\mathcal{N}+2$ redundant variables referred to in (4.30); the three remaining redundant degrees of freedom may be represented by adding arbitrary constants to $\xi_{j}^{\ell}, \eta_{j}^{\ell}$ and $\zeta_{j}^{\ell}$, as we shall see.

\subsection{Elimination of Redundant Plaquette-Flux Variables}

The lattice variables in the Bloch wave number space can be readily expressed in terms of the $2 \mathcal{N}+1$ physical momentum-related ones, given by (4.20)-(4.22). However, for applications to confined configurations, such as glueballs, etc., the use of lattice coordinate space variables gives a more direct physical description. In this section, we give an explicit procedure for the elimination of $\mathcal{N}+2$ redundant variables among the $3 \mathcal{N}$ plaquette-fluxes $\xi_{j}^{\ell}, \eta_{j}^{\ell}$ and $\zeta_{j}^{\ell}$, in accordance with (4.30). Again, the group superscript $\ell$ is kept fixed throughout this section.

In the dual lattice construct a "tree" called $T$, by connecting arbitrarily $\mathcal{N}-1$ links (without any closed cycles). Start from one of the end lattice sites of $T$ and label it 0 . The tree connects 0 to another lattice site, say 1 , through one of its links. Continue this way to move along the tree through all its links. Since each new link leads to a new lattice site, the $\mathcal{N}-1$ links of $T$ must lead from the site 0 to $\mathcal{N}-1$ other lattice sites; i.e., the tree $T$ connects all $\mathcal{N}$ sites in the dual lattice.

Embed the dual lattice $L_{D}$ within the original cubic lattice $L$; each of the links in $L_{D}$ penetrates through a plaquette in $L$. Assign arbitrarily a distribution of $3 \mathcal{N}$ plaquette-fluxes $\left(\xi_{j}^{\ell}, \eta_{j}^{\ell}\right.$, and $\left.\zeta_{j}^{\ell}\right)$ in $L$, each corresponding to a link-current in $L_{D}$. Again, start from the end site 0 of $T$ in $L_{D}$; by assigning the potential difference $\chi_{0}^{\ell}-\chi_{1}^{\ell}$ between the sites 0 and 1 to be the negative of 
the link current along $\overline{01}$, we can change that link-current (and therefore also the corresponding plaquette-flux in $L$ ) to zero through transformation (4.29). Proceed the same way along the tree $T$; since there are $\mathcal{N}-1$ arbitrary potential differences between its $\mathcal{N}$ lattice sites, we can transform all the link-currents along $T$ to become zero in $L_{D}$; i.e., all the corresponding

$$
\mathcal{N}-1
$$

plaquette-fluxes in $L$ can be set to zero through (4.29), without affecting any of the link-fluxes $\left(a_{j}^{\ell}, b_{j}^{\ell}, c_{j}^{\ell}\right)$. Since the lattice sites in $L_{D}$ are all located at the centers of the cubic cells in $L$, the entire tree $T$ does not touch the surfaces of the original lattice $L$. In other words, none of the $\mathcal{N}-1$ plaquette-fluxes that have been set to zero lie on the surfaces of $L$.

Without the boundary conditions, the entire cubic lattice $L$ would have six square surfaces. Take any one of these surfaces, say $S$; it would have four edges. Assign an overall plaquette-flux $I^{\ell}$ flowing along the edges of $S$; because of periodic boundary conditions, we see that the edges facing each other become one, but with $I^{\ell}$ flowing along opposite directions, therefore cancelling each other. Furthermore, the six surfaces of $L$ reduce to three different ones. An overall plaquette-flux on any of these three surfaces produces no link-fluxes. Thus, from the tree $T$, we can add three links in the dual lattice $L_{D}$, with each of the three different surfaces of $L$ penetrated once: Assign zero also to the link-currents along these three additional links in $L_{D}$, therefore making the three corresponding plaquette-fluxes in $L$ also zero. (This is a specific way of eliminating the three remaining redundant degrees of freedom by adding constants to $\xi_{j}^{\ell}, \eta_{j}^{\ell}$ and $\zeta_{j}^{\ell}$, mentioned at the end of Section 4.2.) Together with (4.31), we have succeeded in setting $\mathcal{N}-1+3=\mathcal{N}+2$ plaquette-fluxes to zero, without affecting the link-flux distributions in $L$. This then removes the redundancy mentioned in (4.30). The remaining $2 \mathcal{N}-2$ nonzero plaquette-fluxes $\left(\xi_{j}^{\ell}, \eta_{j}^{\ell}, \zeta_{j}^{\ell}\right)$ and the three constant $A_{a}^{\ell}$ of (4.21) give the $2 \mathcal{N}+1$ independent lattice coordinate space variables.

An alternative procedure to eliminate the redundant lattice variables in the coordinate space is given in Appendix B. 


\section{LATTICE COUPLING CONSTANT}

\subsection{Lattice $Q C D$ Hamiltonian}

For the continuum QCD, the Hamiltonian $\mathcal{H}$, (4.3)-(4.4) in the Coulomb gauge, is written in terms of $\overrightarrow{\mathcal{A}}^{\ell}(\vec{r}, t)$ and its conjugate momentum $\overrightarrow{\mathcal{P}}^{\ell}(\vec{r}, t)$, which satisfy (4.1) and (4.2). In our lattice formulation, $\overrightarrow{\mathcal{A}}^{\ell}(\vec{r}, t)$ and $\overrightarrow{\mathcal{P}}^{\ell}(\vec{r}, t)$ are replaced by $\vec{A}^{\ell}(\vec{r}, t)$ and $\vec{\Pi}^{\ell}(\vec{r}, t)$, given by (4.10)-(4.11), which denote the same field operators, but restricted to the $0^{\text {th }}$-band Bloch functions. At a given lattice size $\ell$ and for a finite total volume $\Omega=(N \ell)^{3}$, the system has only a finite number of variables, as discussed in the previous section. Correspondingly, the continuum Hamiltonian $\mathcal{H}$ reduces to the lattice Hamiltonian

$$
H=\frac{1}{2} \int\left[J^{-1} \Pi_{a}^{\ell} J \Pi_{a}^{\ell}+B_{a}^{\ell} B_{a}^{\ell}\right] d^{3} r+H_{\mathrm{Coul}}
$$

where, in place of (4.4)-(4.8), the lattice Coulomb interaction is

$$
\begin{gathered}
H_{\text {Coul }}=\frac{1}{2} g_{\ell}^{2} J^{-1} \int \sigma_{L}^{\ell}(\vec{r})\left(\ell, \vec{r}\left|\left(\nabla_{a} D_{a}\right)^{-1}\left(-\nabla^{2}\right)\left(\nabla_{b} D_{b}\right)^{-1}\right| \ell^{\prime}, \vec{r}^{\prime}\right) \\
\cdot J \sigma_{L}^{\ell^{\prime}}\left(\vec{r}^{\prime}\right) d^{3} r d^{3} r^{\prime},
\end{gathered}
$$

with the charge density now being

$$
\sigma_{L}^{\ell}=f^{\ell m n} A_{a}^{m} \Pi_{a}^{n}
$$

the color magnetic field $B_{a}^{\ell}$ given by

$$
\epsilon_{a b c} B_{c}^{\ell}=\nabla_{a} A_{b}^{\ell}-\nabla_{b} A_{a}^{\ell}+g_{\ell} f^{\ell m n} A_{a}^{m} A_{b}^{n}
$$

$D_{a}$ denoting the covariant derivative matrix whose elements are

$$
D_{a}^{\ell m}=\delta^{\ell m} \nabla_{a}-g_{\ell} f^{\ell m n} A_{a}^{n}
$$

and $J$ the $0^{t h}$-band Faddeev-Popov determinant (Jacobian)

$$
J=\operatorname{det}\left|\nabla_{a} D_{a}\right|
$$

As in (4.1)-(4.8), all superscripts $\ell, m, n$ refer to the group indices, from 1 to $n^{2}-1$ for $S U(n)$ and all repeated indices are summed over. (Notice that the subscript $\ell$ in $g_{\ell}$ denotes "lattice".) 
The relation between the continuum and the lattice Hamiltonians $\mathcal{H}$ and $H$ may also be summarized as follows: Regard the continuum QCD Hamiltonian $\mathcal{H}$ as a functional $\mathcal{F}$ of $\overrightarrow{\mathcal{A}}^{\ell}, \overrightarrow{\mathcal{P}}^{\ell}$ and the bare coupling $g_{0}$. We write

$$
\mathcal{H}=\mathcal{F}\left(\overrightarrow{\mathcal{A}}^{\ell}, \overrightarrow{\mathcal{P}}^{\ell}, g_{0}\right)
$$

in accordance with (4.3)-(4.8). The lattice QCD Hamiltonian $H$ denotes the same functional $\mathcal{F}$, but with $\overrightarrow{\mathcal{A}^{a}}, \overrightarrow{\mathcal{P}}^{a}$ and $g_{0}$ replaced by $\vec{A}^{a}, \vec{\Pi}^{a}$ and $g_{\ell}$; i.e.,

$$
H=\mathcal{F}\left(\vec{A}^{a}, \vec{\Pi}^{a}, g_{\ell}\right) .
$$

Since the continuum QCD action is noncompact, so is the lattice QCD action. Because $H$ consists of only a finite degree of freedom, the lattice coupling constant $g_{\ell}$ does not have to be renormalized, unlike (4.9) for the continuum case. In the following, we shall examine the relation between the lattice coupling $g_{\ell}$, the continuum bare coupling $g_{0}$ and its renormalization.

\subsection{Bare and Renormalized Continuum Coupling Constants}

A convenient and often used definition of the renormalized coupling in the continuum case is to express it in terms of the interaction energy associated with two external static color charges $e_{1}^{\ell}$ and $e_{2}^{\ell}$, positioned at a distance $R$ apart: Replace the continuum charge density (4.5) by

$$
\sigma^{\ell}(\vec{r})=f^{\ell m n} \mathcal{A}_{a}^{m}(\vec{r}) \mathcal{P}_{a}^{n}(\vec{r})+\sigma_{\text {ext }}^{\ell}(\vec{r})
$$

where

$$
\sigma_{\text {ext }}^{\ell}(\vec{r})=e_{1}^{\ell} \delta^{3}\left(\vec{r}-\vec{R}_{1}\right)+e_{2}^{\ell} \delta^{3}\left(\vec{r}-\vec{R}_{2}\right),
$$

with $e_{1}^{\ell}$ and $e_{2}^{\ell}$ located at $\vec{R}_{1}$ and $\vec{R}_{2}$, and both being $\left(n^{2}-1\right)$-dimensional vectors in the group space. Let $E_{12}$ denote the part of the energy that is proportional to the product $e_{1}^{\ell}$ times $e_{2}^{\ell}$. Write

$$
E_{12} \equiv \frac{g_{R}^{2}}{4 \pi} \frac{e_{1}^{\ell} e_{2}^{\ell}}{R}
$$

where $R=\left|\vec{R}_{1}-\vec{R}_{2}\right|$. At a given $R$, the coefficient $g_{R}^{2}$ can serve as the square of the renormalized coupling. In perturbation series ${ }^{8}$ (assuming $g_{R}^{2}<<1$ ) 


$$
g_{R}^{2}=g_{0}^{2}\left[1+\frac{11 n g_{0}^{2}}{24 \pi^{2}}(\ln \Lambda R+\gamma)\right]+O\left(g_{0}^{6}\right)
$$

where $\Lambda$ is the ultraviolet momentum cutoff and $\gamma=0.5772157$ is the Euler constant. (The reason for separating out the constant $\gamma$ from $\ln \Lambda R$ is connected with (5.64) below.) The inverse relation of (5.12) may be written as

$$
g_{0}^{2}=g_{R}^{2}\left[1+\frac{11 n g_{R}^{2}}{24 \pi^{2}}(\ln \Lambda R+\gamma)\right]^{-1}+O\left(g_{R}^{6}\right) .
$$

Thus, at two distances $R$ and $R^{\prime}$, to the same perturbative order the corresponding renormalized couplings $g_{R}$ and $g_{R^{\prime}}$ satisfy the familiar asymptotic freedom relation ${ }^{8}$

$$
\frac{1}{g_{R^{\prime}}^{2}}-\frac{1}{g_{R}^{2}}=\frac{11 n}{24 \pi^{2}} \ln R / R^{\prime} .
$$

It follows then, for $R^{\prime}<R, g_{R^{\prime}}^{2}<g_{R}^{2}$; in particular, when $R^{\prime} / R \rightarrow 0, g_{R^{\prime}}^{2} \rightarrow 0$.

\subsection{Lattice Coupling $g_{\ell}$ As the Renormalized Continuum Coupling Constant}

Next, we consider the lattice Hamiltonian (5.1)-(5.2). Replace the lattice charge density (5.3) by

$$
\sigma_{L}^{\ell}(\vec{r})=f^{\ell m n} A_{a}^{m}(\vec{r}) \Pi_{a}^{n}(\vec{r})+\sigma_{\text {ext }}^{\ell}(\vec{r})
$$

with $\sigma_{\text {ext }}^{\ell}(\vec{r})$ given by the external charge density identical to (5.10), and then evaluate the same interaction energy $E_{12}$ that is proportional to $e_{1}^{\ell}$ times $e_{2}^{\ell}$. Write the lattice result as

$$
E_{12}=\frac{\left(g_{R}^{2}\right)_{L}}{4 \pi} \frac{e_{1}^{\ell} e_{2}^{\ell}}{R}
$$

where, unlike (5.11)-(5.12), $\left(g_{R}^{2}\right)_{L}$ is a finite function of the lattice coupling $g_{\ell}^{2}$. Because $H$ does not have the symmetry of the continuum, but only that of a cubic lattice, $\left(g_{R}^{2}\right)_{L}$ depends also on $\vec{R}_{1}, \vec{R}_{2}$ and the lattice size $\ell$. We may select any $\vec{R}_{1}$ and $\vec{R}_{2}$, and set

$$
\left(g_{R}^{2}\right)_{L}=g_{R}^{2}
$$


i.e., we choose the lattice coupling $g_{\ell}$ so that the interaction energy $E_{12}\left(\vec{R}_{1}, \vec{R}_{2}\right)$ calculated from the continuum Hamiltonian $\mathcal{H}$ and that from its $0^{\text {th }}$-band approximation $H$ are exactly equal for a particular pair of position vectors $\vec{R}_{1}$ and $\vec{R}_{2}$. Because of the cubic lattice symmetry, this equality extends to all pairs of $\vec{R}_{1}, \vec{R}_{2}$ which can be reached from the selected ones through a lattice translation and a cubic rotation.

The precise meaning of this equality can be stated in an alternative way: Express the continuum coupling constant renormalization formula (4.9) in the form

$$
g_{0}=g_{\ell}+\delta g
$$

i.e., instead of using (5.13), regard the lattice coupling $g_{\ell}$ as the renormalized coupling for the continuum case. Expand $\overrightarrow{\mathcal{A}}^{\ell}$ and $\overrightarrow{\mathcal{P}}^{\ell}$ in terms of the complete set of Bloch functions $\left\{\vec{F}_{n}^{t}(\vec{K} \mid \vec{r})\right\}$, given by (3.72)-(3.73). Decompose

$$
\overrightarrow{\mathcal{A}}^{\ell}=\vec{A}^{\ell}+\delta \overrightarrow{\mathcal{A}}^{\ell} \quad \text { and } \quad \overrightarrow{\mathcal{P}}^{\ell}=\vec{\Pi}^{\ell}+\delta \overrightarrow{\mathcal{P}}^{\ell}
$$

where $\delta \overrightarrow{\mathcal{A}}^{\ell}$ and $\delta \overrightarrow{\mathcal{P}}^{\ell}$ consist of all terms that depend on $\vec{n} \neq 0$-band Bloch functions. Substituting (5.18)-(5.19) into the functional (5.7), we can express the continuum $\mathcal{H}$ as a sum

$$
\mathcal{H}=H+\delta \mathcal{H},
$$

where $\delta \mathcal{H}$ contains all terms that depend on $\delta \overrightarrow{\mathcal{A}}^{\ell}, \delta \overrightarrow{\mathcal{P}}^{\ell}$ and $\delta g$. The condition for determining $\delta g$ is: For $e_{1}^{\ell}$ and $e_{2}^{\ell}$ located at the selected pair of position vectors $\vec{R}_{1}$ and $\vec{R}_{2}$, the interaction energy $E_{12}$ calculated from $\mathcal{H}$ equals that using $H$; i.e., $\delta \mathcal{H}$ gives no correction. At different $\vec{R}_{1}$ and $\vec{R}_{2}$ there will be, in general, a correction due to $\delta \mathcal{H}$.

Because of asymptotic freedom, there is a wide range of $R$ and lattice size $\ell$, for which the equality (5.17) can lead to $g_{\ell}^{2}<<1$, in which case corrections due to $\delta \mathcal{H}$ can be computed perturbatively in powers of $g_{\ell}^{2}$. In the present noncompact lattice formulation, there is no need to take the limit $\ell \rightarrow 0$, since the continuum solution is independent of the lattice size $\ell$.

In the following, we assume that $g_{\ell}^{2}$ is indeed small; this enables us to relate perturbatively $g_{\ell}^{2}$ with $g_{R}^{2}$, defined by (5.11). It is convenient to write 


$$
g_{R}^{2}=g_{\ell}^{2}\left[1+\frac{11 n}{24 \pi^{2}} g_{\ell}^{2}\left(\ln \frac{R}{\ell}+\gamma+\lambda+\delta\right)\right]+O\left(g_{\ell}^{6}\right)
$$

where, besides the Euler constant $\gamma$, there is another numerical constant $\lambda$ and a function

$$
\delta=\delta\left(\ell, \vec{R}_{1}, \vec{R}_{2}\right)
$$

which satisfies

$$
\lim _{\ell \rightarrow 0} \delta\left(\ell, \vec{R}_{1}, \vec{R}_{2}\right)=0
$$

Accordingly, (5.18) becomes

$$
g_{0}^{2}=g_{\ell}^{2}-\frac{11 n}{24 \pi^{2}} g_{\ell}^{4}(\ln \Lambda \ell-\lambda-\delta)+O\left(g_{\ell}^{6}\right)
$$

In the rest of the paper, we shall evaluate the constant $\lambda$ and derive the formula for $\delta$. As we shall see,

$$
\lambda=3.3559 .
$$

The expression for $\delta$ is given in Appendix C (see (C.33)).

\subsection{Power Series Expansion of the Lattice Hamiltonian}

In the $0^{\text {th }}$-band approximation, the Hamiltonian (5.1)-(5.2) can be expanded into a power series of the lattice coupling constant $g_{\ell}$ :

$$
H=H_{0}+H_{\mathrm{int}}
$$

where $H_{0}$ is $g_{\ell}$ independent and

$$
H_{\mathrm{int}}=g_{\ell} H_{1}+g_{\ell}^{2} H_{2}+g_{\ell}^{3} H_{3}+g_{\ell}^{4} H_{4}+\cdots .
$$

The field $A_{a}^{\ell}(\vec{r})$ and its conjugate momentum $\Pi_{a}^{\ell}(\vec{r})$ are given by (4.10) and (4.11). At any given time $t$, they can also be written in terms of the creation and annihilation operators:

$$
A_{a}^{\ell}(\vec{r}, t)=\sum_{\vec{K}, t} \frac{1}{\sqrt{2 \omega_{t}(\vec{K})}}\left[a_{t}^{\ell}(\vec{K}) \hat{\epsilon}_{t}(\vec{K})_{a} F_{a}(\vec{K} \mid \vec{r})+a_{t}^{\ell}(\vec{K})^{\dagger} \hat{\epsilon}_{t}(\vec{K})_{a} F_{a}(\vec{K} \mid \vec{r})^{*}\right]
$$


and

$\Pi_{a}^{\ell}(\vec{r}, t)=-i \sum_{\vec{K}, t} \sqrt{\frac{\omega_{t}(\vec{K})}{2}}\left[a_{t}^{\ell}(\vec{K}) \hat{\epsilon}_{t}(\vec{K})_{a} F_{a}(\vec{K} \mid \vec{r})-a_{t}^{\ell}(\vec{K})^{\dagger} \hat{\epsilon}_{t}(\vec{K})_{a} F_{a}(\vec{K} \mid \vec{r})^{*}\right]$

where $\hat{\epsilon}_{t}(\vec{K})$ and $\omega_{t}(\vec{K})$, with $t=1,2$ (or,+- ) are given by (3.47) and (3.56), $a_{t}^{\ell}(\vec{K})$ and $a_{t}^{\ell}(\vec{K})^{\dagger}$ satisfy the usual commutation relation

$$
\left[a_{t}^{\ell}(\vec{K}), a_{t^{\prime}}^{\ell^{\prime}}\left(\vec{K}^{\prime}\right)^{\dagger}\right]=\delta^{\ell \ell^{\prime}} \delta_{t t^{\prime}} \delta_{\vec{K}, \vec{K}^{\prime}} .
$$

As before, $\vec{K}$ and $\vec{K}^{\prime}$ are Bloch wave number vectors within the Brillouin zone. The operators $a_{t}^{\ell}(\vec{K})$ and $a_{t}^{\ell}(\vec{K})^{\dagger}$ are related to $q_{a}^{\ell}(\vec{K})$ and $p_{a}^{\ell}(\vec{K})$ of $(4.10)$ and (4.11) by

$$
q_{a}^{\ell}(\vec{K})=\sum_{t} \frac{1}{\sqrt{2 \omega_{t}(\vec{K})}}\left[a_{t}^{\ell}(\vec{K}) \hat{\epsilon}_{t}(\vec{K})_{a}+a_{t}^{\ell}(-\vec{K})^{\dagger} \hat{\epsilon}_{t}(-\vec{K})_{a}\right]
$$

and

$$
p_{a}^{\ell}(-\vec{K})=-i \sum_{t} \sqrt{\frac{\omega_{t}(\vec{K})}{2}}\left[a_{t}^{\ell}(\vec{K}) \hat{\epsilon}_{t}(\vec{K})_{a}-a_{t}^{\ell}(-\vec{K})^{\dagger} \hat{\epsilon}_{t}(-\vec{K})_{a}\right] .
$$

In terms of these annihilation and creation operators, the zeroth-order Hamiltonian in (5.27) becomes, apart from an additive constant which may be set to zero,

$$
H_{0}=\sum_{\vec{K}, t, \ell} \omega_{t}(\vec{K}) a_{t}^{\ell}(\vec{K})^{\dagger} a_{t}^{\ell}(\vec{K}) .
$$

The eigenvalues of $H_{0}$ are

$$
E_{0}(N)=\sum_{\vec{K}, t, \ell} N_{\vec{K}, t}^{\ell} \omega_{t}(\vec{K})
$$

where $N_{\vec{K}, t}^{\ell}=0,1,2, \cdots$ is the eigenvalue of the occupation-number operator $a_{t}^{\ell}(\vec{K})^{\dagger} a_{t}^{\ell}(\vec{K})$. Let the corresponding eigenstates be $|N\rangle$, with $|0\rangle$ denoting the ground state of $H_{0}$. Thus, we have 


$$
a_{t}^{\ell}(\vec{K}) \mid 0>=0
$$

and $E_{0}(0)=0$. To $O\left(g_{\ell}^{2}\right)$, the shift in the ground state energy $E_{0}\left(\vec{R}_{1}, \vec{R}_{2}\right)$ due to $H_{\mathrm{int}}$ of (5.26)-(5.27) is given by the familiar perturbation formula

$$
E_{0}\left(\vec{R}_{1}, \vec{R}_{2}\right)=<0\left|H_{\text {int }}\right| 0>-\sum_{N \neq 0} \frac{|<N| H_{\text {int }}|0>|^{2}}{E_{0}(N)}+O\left(H_{\text {int }}^{3}\right)
$$

the interaction energy $E_{12}$, defined by (5.14), refers to the part of $E_{0}\left(\vec{R}_{1}, \vec{R}_{2}\right)$ which is proportional to the product $e_{1}^{\ell} e_{2}^{\ell}$ and corresponds to the following difference:

$$
E_{12}=E_{0}\left(\vec{R}_{1}, \vec{R}_{2}\right)-\lim _{R \rightarrow \infty} E_{0}\left(\vec{R}_{1}, \vec{R}_{2}\right)
$$

where $R=\left|\vec{R}_{2}-\vec{R}_{1}\right|$, as before.

In the following, $E_{12}$ will be expanded in powers of $H_{\text {int }}$ :

$$
E_{12}=E_{12}^{(i)}+E_{12}^{(i i)}+\cdots
$$

where $E_{12}^{(i)}$ is first-order in $H_{\mathrm{int}}$ and $E_{12}^{(i i)}$ second order in $H_{\mathrm{int}}$. As we shall see, $E_{12}^{(i)}$ contains $O\left(g_{\ell}^{2}\right), O\left(g_{\ell}^{4}\right)$ and higher order terms; likewise $E_{12}^{(i i)}$ contains $O\left(g_{\ell}^{4}\right)$ and higher-order terms. In this paper, we shall calculate $E_{12}$ only up to $O\left(g_{\ell}^{4}\right)$.

\subsection{First Order in $H_{\mathrm{int}}$}

To first order in $H_{\mathrm{int}},(5.35)$ reduces to

$$
E_{0}\left(\vec{R}_{1}, \vec{R}_{2}\right)=<0\left|H_{\text {int }}\right| 0>
$$

in which only the part proportional to $e_{1}^{\ell} e_{2}^{\ell}$ gives $E_{12}^{(i)}$. Substituting the power series expansion (5.27) into (5.38), we see that since all odd $g_{\ell}$-power terms carry odd numbers of gauge field operators, whose vacuum expectation values are zero, the result is an even function of $g_{\ell}$. Because $E_{12}$ is proportional to $e_{1}^{\ell} e_{2}^{\ell}$, for our purpose we can equate $H_{\text {int }}=H_{\text {Coul }}$, which is given by (5.2). Up to $O\left(g_{\ell}^{4}\right)$, we can also set the Jacobian $J=1$. In power series of $g_{\ell}$, we expand 


$$
\begin{aligned}
\left(\nabla_{a} D_{a}\right)^{-1}\left(-\nabla^{2}\right)\left(\nabla_{b} D_{b}\right)^{-1}= & -\nabla^{-2}+2 g_{\ell} \nabla^{-2} \nabla_{a} A_{a} \nabla^{-2} \\
& -3 g_{\ell}^{2} \nabla^{-2} \nabla_{a} A_{a} \nabla^{-2} \nabla_{b} A_{b} \nabla^{-2}+\cdots
\end{aligned}
$$

where $A_{a}$ denotes the $\left(n^{2}-1\right) \times\left(n^{2}-1\right)$ matrix whose $\left(\ell, \ell^{\prime}\right)$ element is related to the $0^{\text {th }}$-band gauge field $A_{a}^{m}(\vec{r})$ by

$$
\left(A_{a}\right)^{\ell \ell^{\prime}}=f^{\ell m \ell^{\prime}} A_{a}^{m} .
$$

Hence, we find

$$
E_{12}^{(i)}=g_{\ell}^{2} \frac{e_{1}^{\ell} e_{2}^{\ell}}{4 \pi R}+g_{\ell}^{4} \mathcal{E}^{(i)}+O\left(g_{\ell}^{6}\right)
$$

where

$$
\mathcal{E}^{(i)}=-3 e_{1}^{\ell} e_{2}^{\ell^{\prime}}<0\left|\left(\ell, \vec{R}_{1}\left|\nabla^{-2} \nabla_{a} A_{a} \nabla^{-2} \nabla_{b} A_{b} \nabla^{-2}\right| \ell^{\prime}, \vec{R}_{2}\right)\right| 0>.
$$

From (5.28) and (5.34), it follows that

$<0\left|A_{a}^{\ell}(\vec{r}) A_{b}^{m}\left(\vec{r}^{\prime}\right)\right| 0>=\delta^{\ell m} \sum_{\vec{K}, t}\left[2 \omega_{t}(\vec{K})\right]^{-1} \hat{\epsilon}_{t}(\vec{K})_{a} \hat{\epsilon}_{t}(\vec{K})_{b} F_{a}(\vec{K} \mid \vec{r}) F_{b}\left(\vec{K} \mid \vec{r}^{\prime}\right)^{*}$

where, as before, $F_{a}(\vec{K} \mid \vec{r})$ is the $0^{t h}$-band Bloch function, given by (3.38). In (5.43), the repeated subscripts are not to be summed over. Using the familiar expressions

$$
\begin{aligned}
\left(\vec{R}_{1}\left|\nabla^{-2} \nabla_{a}\right| \vec{r}\right) & =\int-\frac{d^{3} p}{(2 \pi)^{3}} \frac{i p_{a}}{p^{2}} e^{i \vec{p} \cdot\left(\vec{R}_{1}-\vec{r}\right)}, \\
\left(\vec{r}^{\prime}\left|\nabla_{b} \nabla^{-2}\right| \vec{R}_{2}\right) & =\int-\frac{d^{3} p^{\prime}}{(2 \pi)^{3}} \frac{i p_{b}^{\prime}}{p^{\prime 2}} e^{i \vec{p}^{\prime} \cdot\left(\vec{r}^{\prime}-\vec{R}_{2}\right)}
\end{aligned}
$$

and

$$
\left(\vec{r}\left|\nabla^{-2}\right| \vec{r}^{\prime}\right)=\int-\frac{d^{3} q}{(2 \pi)^{3}} \frac{1}{q^{2}} e^{i \vec{q} \cdot\left(\vec{r}-\vec{r}^{\prime}\right)}
$$

with $p, p^{\prime}$ and $q$ denoting the magnitudes of $\vec{p}, \vec{p}^{\prime}$ and $\vec{q}$, and combining these with (5.42)-(5.43), we derive ${ }^{9}$

$$
\begin{aligned}
\mathcal{E}^{(i)}=3 n e_{1}^{\ell} e_{2}^{\ell} \sum \int e^{i \phi} & \frac{p_{a} p_{b}^{\prime}}{p^{2} p^{\prime 2} q^{2}}\left[2 \omega_{t}(\vec{K})\right]^{-1} \hat{\epsilon}_{t}(\vec{K})_{a} \hat{\epsilon}_{t}(\vec{K})_{b} \\
& \times F_{a}(\vec{K} \mid \vec{r}) F_{b}\left(\vec{K} \mid \vec{r}^{\prime}\right)^{*} d^{3} r d^{3} r^{\prime}
\end{aligned}
$$


where

$$
\phi=\vec{p} \cdot\left(\vec{r}-\vec{R}_{1}\right)+\vec{q} \cdot\left(\vec{r}^{\prime}-\vec{r}\right)+\vec{p}^{\prime}\left(\vec{R}_{2}-\vec{r}^{\prime}\right)
$$

and the summation extends over the polarization index $t=1,2, \vec{K}$ within the Brillouin zone, $\vec{p}, \vec{p}^{\prime}$ and $\vec{q}$ over all wave numbers, and the subscripts $a, b=x, y$ and $z$.

Because $e^{-i \vec{K} \cdot \vec{r}}$ times the Bloch wave function $F_{a}(\vec{K} \mid \vec{r})$ has the periodicity of the lattice, the integrand in (5.45) consists of a periodic function in $\vec{r}$ and $\vec{r}^{\prime}$ multiplied by a phase factor $e^{i \phi+i \vec{K} \cdot\left(\vec{r}-\vec{r}^{\prime}\right)}$. Resolve the phase into a sum of terms

$$
\phi+\vec{K} \cdot\left(\vec{r}-\vec{r}^{\prime}\right)=(\vec{p}-\vec{q}+\vec{K}) \cdot \vec{r}-\left(\vec{p}^{\prime}-\vec{q}+\vec{K}\right) \cdot \vec{r}^{\prime}+\left(\vec{p}^{\prime} \cdot \vec{R}_{2}-\vec{p} \cdot \vec{R}_{1}\right) .
$$

The integration over $\vec{r}$ and $\vec{r}^{\prime}$ gives

$$
\vec{p}-\vec{q}+\vec{K}=2 \pi \vec{m}_{1} / \ell
$$

and

$$
\vec{p}^{\prime}-\vec{q}+\vec{K}=2 \pi \vec{m}_{2} / \ell
$$

where $\vec{m}_{1}$ and $\vec{m}_{2}$ are three-dimensional vectors whose components are all integers. Introduce

$$
\begin{aligned}
\vec{k} & =\frac{1}{2}\left(\vec{p}^{\prime}+\vec{p}\right), \\
\vec{R}_{\mathrm{cm}} & =\frac{1}{2}\left(\vec{R}_{2}+\vec{R}_{1}\right)
\end{aligned}
$$

and

$$
\vec{R}=\vec{R}_{2}-\vec{R}_{1}
$$

The last term in (5.47) can be written as

$$
\vec{p}^{\prime} \cdot \vec{R}_{2}-\vec{p} \cdot \vec{R}_{1}=\frac{2 \pi}{\ell}\left(\vec{m}_{2}-\vec{m}_{1}\right) \cdot \vec{R}_{\mathrm{cm}}+\vec{k} \cdot \vec{R} .
$$

Consequently, $\mathcal{E}^{(i)}$ is a periodic function in $\vec{R}_{\mathrm{cm}}$ with the periodicity of the lattice cell. 
By using (5.48)-(5.49), we can convert the sum over $\vec{p}, \vec{p}^{\prime}$ and $\vec{q}$ into an integration over $\vec{k}$ times a sum over $\vec{m}_{1}$ and $\vec{m}_{2}$; this leads to

$$
\mathcal{E}^{(i)}=3 n e_{1}^{\ell} e_{2}^{\ell} \int \frac{d^{3} k}{(2 \pi)^{3}} \frac{e^{i \vec{k} \cdot \vec{R}}}{k^{2}} I(\vec{k})
$$

where $I(\vec{k})$ is a dimensionless function, given by

$$
I(\vec{k})=\sum_{\vec{m}_{1}, \vec{m}_{2}} \int_{B} \frac{d^{3} K}{(2 \pi)^{3}} f_{\vec{m}_{1} \vec{m}_{2}}(\vec{K}, \vec{k}) e^{i \frac{2 \pi}{\ell}\left(\vec{m}_{2}-\vec{m}_{1}\right) \cdot \vec{R}_{\mathrm{cm}}}
$$

with the $\vec{k}$-integration over the entire continuum momentum space, but the $\vec{K}$-integration only within the Brillouin zone as indicated by the subscript $B$,

$$
\begin{aligned}
f_{\vec{m}_{1} \vec{m}_{2}}(\vec{K}, \vec{k})=k^{2} \sum_{t, a, b} \frac{\Omega^{\frac{1}{2}} \mathcal{C}_{a}\left(\vec{\theta} \mid-\vec{m}_{1}\right) \Omega^{\frac{1}{2}} \mathcal{C}_{b}\left(\vec{\theta} \mid-\vec{m}_{2}\right) \hat{\epsilon}_{t}(\vec{K})_{a} \hat{\epsilon}_{t}(\vec{K})_{b}}{2 \omega_{t}(\vec{K})\left(\vec{k}+\vec{K}-\frac{\vec{m}_{1}+\vec{m}_{2}}{\ell} \pi\right)^{2}} \\
\times \frac{\left(\vec{k}+\frac{\vec{m}_{1}-\vec{m}_{2}}{\ell} \pi\right)_{a}\left(\vec{k}-\frac{\vec{m}_{1}-\vec{m}_{2}}{\ell} \pi\right)_{b}}{\left(\vec{k}+\frac{\vec{m}_{1}-\vec{m}_{2}}{\ell} \pi\right)^{2}\left(\vec{k}-\frac{\vec{m}_{1}-\vec{m}_{2}}{\ell} \pi\right)^{2}},
\end{aligned}
$$

and $\Omega^{\frac{1}{2}} \mathcal{C}_{a}(\vec{\theta} \mid \vec{m})$ given by (3.67) and (3.40), which is both dimensionless and independent of $\Omega$. From (5.52), one sees directly that $I(\vec{k})$ and therefore $\mathcal{E}^{(i)}$ are both periodic in $\vec{R}_{\mathrm{cm}}$.

To evaluate the contribution of $\mathcal{E}^{(i)}$ to the constant $\lambda$ in (5.21), we examine the limiting case $\ell / R \rightarrow 0$ and call the result $\lambda^{(i)}$. The difference between the limiting result and that for an arbitrary nonzero $\ell / R$ gives the corresponding contribution to the remainder $\delta\left(\ell, \vec{R}_{1}, \vec{R}_{2}\right)$. When $\ell / R \rightarrow 0$, the integral (5.51) is dominated by the region $k \ell=O\left(R^{-1}\right)$. Furthermore, because of the rapid oscillatory phase factor $\exp \left[i \frac{2 \pi}{\ell}\left(\vec{m}_{1}-\vec{m}_{2}\right) \cdot \vec{R}_{\mathrm{cm}}\right]$ in $(5.52)$, we need only consider the special case

$$
\vec{m}_{1}=\vec{m}_{2}=\vec{m}
$$

as a result, $I(\vec{k})$ becomes independent of $\vec{R}_{\mathrm{cm}}$. From (5.53), one can readily verify that as $\vec{k}$ and $\vec{K}$ both $\rightarrow 0$, the sum (5.52) over $\vec{m}$ is controlled by the single term $\vec{m}_{1}=\vec{m}_{2}=0$, for which the limiting behavior of the corresponding $f_{\vec{m}_{1} \vec{m}_{2}}(\vec{K}, \vec{k})$ is given by the continuum function $f_{c}$ : 


$$
f_{00}(\vec{K}, \vec{k}) \rightarrow f_{c}(\vec{K}, \vec{k}) \equiv \frac{1}{2 K(\vec{k}+\vec{K})^{2}} \sum_{t} \frac{\left[\vec{k} \cdot \hat{\epsilon}_{t}(\vec{K})\right]^{2}}{k^{2}}
$$

where $K$ and $k$ refer to the magnitudes of $\vec{K}$ and $\vec{k}$, as before, and the sum over the polarization index $t$ yields

$$
\sum_{t} \hat{\epsilon}_{t}(\vec{K})_{a} \hat{\epsilon}_{t}(\vec{K})_{b}=\delta_{a b}-\frac{K_{a} K_{b}}{K^{2}}
$$

To facilitate the integration (5.52), we decompose $I(\vec{k})$ into a sum of two terms:

$$
I(\vec{k})=I_{c}(\vec{k})+I^{\prime}(\vec{k})
$$

where

$$
I_{c}(\vec{k})=\int_{B} \frac{d^{3} K}{(2 \pi)^{3}} f_{c}(\vec{K}, \vec{k})
$$

and, on account of (5.54),

$$
I^{\prime}(\vec{k})=\int_{B} \frac{d^{3} K}{(2 \pi)^{3}}\left[\sum_{m} f_{\vec{m}, \vec{m}}(\vec{K}, \vec{k})-f_{c}(\vec{K}, \vec{k})\right] .
$$

When $\ell / R \rightarrow 0$, the integration $I_{c}(\vec{k})$ can be evaluated analytically. As shown in Appendix $\mathrm{C}$, the result is

$$
I_{c}(\vec{k})=\frac{1}{6 \pi^{2}}\left(-\ln k \ell+\lambda_{c}\right)
$$

in which the constant $\lambda_{c}$ is

$$
\lambda_{c}=\frac{4}{3}+\ln \pi-\frac{6}{\pi} \int_{0}^{1} d x \frac{\ln \left(x^{2}+2\right)}{\left(x^{2}+1\right) \sqrt{x^{2}+2}}=1.67040 .
$$

The second term $I^{\prime}(\vec{k})$ in (5.57) is well-behaved at small $\vec{k}$; its value at $\vec{k}=0$ can be written as

$$
I^{\prime}(0)=\frac{1}{6 \pi^{2}} \lambda^{\prime}
$$


with the constant $\lambda^{\prime}$ given by

$$
\lambda^{\prime}=-0.1496
$$

By using

$$
\int \frac{d^{3} k}{(2 \pi)^{3}} \frac{1}{k^{2}}(\ln k \ell) e^{i \vec{k} \cdot \vec{R}}=-\frac{1}{4 \pi R}[(\ln R / \ell)+\gamma],
$$

we derive, as $\ell / R \rightarrow 0$,

$$
\mathcal{E}^{(i)}=\frac{e_{1}^{\ell} e_{2}^{\ell}}{4 \pi R}\left(\frac{n}{2 \pi^{2}}\right)\left[(\ln R / \ell)+\gamma+\lambda^{(i)}\right]
$$

where $\gamma$ is the Euler constant and

$$
\lambda^{(i)}=\lambda_{c}+\lambda^{\prime}=1.5208 .
$$

\subsection{Second Order in $H_{\mathrm{int}}$}

In accordance with (5.35)-(5.37),

$$
E_{12}^{(i i)}=-\sum_{N \neq 0} \frac{\left.|<0| H_{\mathrm{int}}|N\rangle\right|^{2}}{E_{0}(N)}+\lim _{R \rightarrow \infty} \sum_{N \neq 0} \frac{\left.|<0| H_{\mathrm{int}}|N\rangle\right|^{2}}{E_{0}(N)}
$$

in which the relevant states $\mid N>$ are those of two gauge quanta, i.e. $|N\rangle=$ $\mid 2>$ with

$$
\left|2>=a_{t_{1}}^{\ell_{1}}\left(\vec{K}_{1}\right)^{\dagger} a_{t_{2}}^{\ell_{2}}\left(\vec{K}_{2}\right)^{\dagger}\right| 0>.
$$

To $O\left(g_{\ell}^{4}\right)$, we can write, similar to (5.41),

$$
E_{12}^{(i i)}=g_{\ell}^{4} \mathcal{E}^{(i i)}+O\left(g_{\ell}^{6}\right)
$$

and set, in (5.67), $H_{\mathrm{int}}=H_{\text {Coul }}$ with $J=1$ and $D_{a}=\nabla_{a}$, so that the relevant part of $H_{\text {Coul }}$ is

$$
H_{\text {Coul }}=g_{\ell}^{2} \int d^{3} r \int d^{3} r^{\prime} \sigma_{A}^{\ell}(\vec{r}) \sigma_{\text {ext }}^{\ell}\left(\vec{r}^{\prime}\right)\left(\vec{r}\left|-\nabla^{-2}\right| \vec{r}^{\prime}\right)
$$


where $\sigma_{A}^{\ell}(\vec{r})=f^{\ell m n} A_{a}^{m}(\vec{r}) \Pi_{a}^{n}(\vec{r})$ is the first term in (5.15). By using (5.28)(5.29), (5.68)-(5.69) and the last equation in (5.44), we obtain

$$
<2\left|H_{\text {Coul }}\right| 0>=g_{\ell}^{2} f^{\ell \ell_{1} \ell_{2}} \sum M_{t_{1} t_{2}}\left(\vec{q} ; \vec{K}_{1}, \vec{K}_{2}\right) q^{-2}\left(e_{1}^{\ell} e^{-i \vec{q} \cdot \vec{R}_{1}}+e_{2}^{\ell} e^{-i \vec{q} \cdot \vec{R}_{2}}\right)
$$

where $\ell_{1}, t_{1}$ and $\ell_{2}, t_{2}$ denote the group and polarization indices of the twogauge quanta in $|2\rangle, \vec{q}$ is the momentum variable in (5.44) for $\nabla^{-2}$, which is related to the Bloch wave numbers $\vec{K}_{1}$ and $\vec{K}_{2}$ of the two quanta by

$$
\vec{q}=\vec{K}_{1}+\vec{K}_{2}+\frac{2 \pi}{\ell} \vec{m}
$$

with the components of $\vec{m}$ all integers. The factor $M_{t_{1} t_{2}}$ is given by

$$
\begin{aligned}
M_{t_{1} t_{2}}\left(\vec{q} ; \vec{K}_{1}, \vec{K}_{2}\right)= & \frac{i\left[\omega_{t_{2}}\left(\vec{K}_{2}\right)-\omega_{t_{1}}\left(\vec{K}_{1}\right)\right]}{2 \sqrt{\omega_{t_{1}}\left(\vec{K}_{1}\right) \omega_{t_{2}}\left(\vec{K}_{2}\right)}} \sum_{a} \hat{\epsilon}_{t_{1}}\left(\vec{K}_{1}\right)_{a} \hat{\epsilon}_{t_{2}}\left(\vec{K}_{2}\right)_{a} \\
& \times \int d^{3} r F_{a}\left(\vec{K}_{1} \mid \vec{r}\right)^{*} F_{a}\left(\vec{K}_{2} \mid \vec{r}\right)^{*} e^{i \vec{q} \cdot \vec{r}} .
\end{aligned}
$$

We observe that when $t_{1}=t_{2}=t$, as $\vec{K}_{1}+\vec{K}_{2} \rightarrow 0$

$$
M_{t_{1} t_{2}}\left(\vec{q} ; \vec{K}_{1}, \vec{K}_{2}\right) \rightarrow 0
$$

for all $\vec{m}$ including 0 , since in this case $\omega_{t_{2}}\left(\vec{K}_{2}\right)-\omega_{t_{1}}\left(\vec{K}_{1}\right)=\omega_{t}\left(\vec{K}_{2}\right)-\omega_{t}\left(\vec{K}_{1}\right) \rightarrow$ 0 . Also, when $t_{1} \neq t_{2}$ but $\vec{m}=0$, as $\vec{K}_{1}+\vec{K}_{2} \rightarrow 0$ we have again

$$
M_{t_{1} t_{2}}\left(\vec{q} ; \vec{K}_{1}, \vec{K}_{2}\right) \rightarrow 0
$$

because

$$
\int d^{3} r F_{a}\left(\vec{K}_{1} \mid \vec{r}\right)^{*} F_{a}\left(\vec{K}_{2} \mid \vec{r}\right)^{*} \rightarrow \int d^{3} r F_{a}\left(\vec{K}_{1} \mid \vec{r}\right)^{*} F_{a}\left(\vec{K}_{1} \mid \vec{r}\right)=1
$$

which makes the subsequent sum over the polarization vectors in (5.73)

$$
\sum_{a} \hat{\epsilon}_{t_{1}}\left(\vec{K}_{1}\right)_{a} \hat{\epsilon}_{t_{2}}\left(-\vec{K}_{1}\right)_{a}=0
$$

Both properties (5.74) and (5.75) will be useful for calculating $\mathcal{E}^{(i i)}$ in the limit $\ell / R \rightarrow 0$. 
Substituting (5.71)-(5.73) into (5.67) and (5.69), we derive

$$
\mathcal{E}^{(i i)}=-n e_{1}^{\ell} e_{2}^{\ell} \sum e^{i \phi^{\prime}} \frac{M_{t_{1} t_{2}}\left(q ; \vec{K}_{1}, \vec{K}_{2}\right)^{*} M_{t_{1} t_{2}}\left(q^{\prime} ; \vec{K}_{1}, \vec{K}_{2}\right)}{\left[\omega_{t_{1}}\left(\vec{K}_{1}\right)+\omega_{t_{2}}\left(\vec{K}_{2}\right)\right] q^{2} q^{\prime 2}}
$$

where

$$
\begin{gathered}
\phi^{\prime}=\vec{q} \cdot \vec{R}_{1}-\vec{q}^{\prime} \cdot \vec{R}_{2}, \\
\vec{q}=\vec{K}_{1}+\vec{K}_{2}+\frac{2 \pi \vec{m}}{\ell}, \\
\vec{q}^{\prime}=\vec{K}_{1}+\vec{K}_{2}+\frac{2 \pi \vec{m}^{\prime}}{\ell},
\end{gathered}
$$

and the sum is over the polarization indices $t_{1}$ and $t_{2}$, the two Bloch wave number vectors $\vec{K}_{1}$ and $\vec{K}_{2}$, as well as the vectors $\vec{m}$ and $\vec{m}^{\prime}$, both having integer components. From (5.49) and (5.77), we have

$$
\phi^{\prime}=\frac{2 \pi}{\ell}\left(\vec{m}-\vec{m}^{\prime}\right) \cdot \vec{R}_{\mathrm{cm}}+\vec{k} \cdot \vec{R}
$$

where

$$
\vec{k}=\vec{K}_{1}+\vec{K}_{2}+\frac{\pi}{\ell}\left(\vec{m}+\vec{m}^{\prime}\right)
$$

Hence, $\mathcal{E}^{(i i)}$ is also a periodic function in $\vec{R}_{\mathrm{cm}}$, the same as $\mathcal{E}^{(i)}$. Likewise, as $\ell \rightarrow 0$, we can set

$$
\vec{m}=\vec{m}^{\prime}
$$

and $\mathcal{E}^{(i i)}$ becomes independent of $\vec{R}_{\mathrm{cm}}$.

To derive the asymptotic behavior of $\mathcal{E}^{(i i)}$ in the limit $\ell / R \rightarrow 0$, we follow steps parallel to those for $\mathcal{E}^{(i)}$. Write

$$
\mathcal{E}^{(i i)}=-n e_{1}^{\ell} e_{2}^{\ell} \int \frac{d^{3} k}{(2 \pi)^{3}} \frac{e^{i \vec{k} \cdot \vec{R}}}{k^{2}} I I(\vec{k})
$$

where

$$
I I(\vec{k})=\int_{B} \frac{d^{3} K}{(2 \pi)^{3}} h(\vec{K}, \vec{k})
$$

with the $\vec{k}$ integration extending over the entire momentum space but the $\vec{K}$ integration only within the Brillouin zone. The function $h$ is given by

$$
h(\vec{K}, \vec{k})=\frac{1}{k^{2}} \sum_{t_{1}, t_{2}} \frac{M_{t_{1} t_{2}}\left(\vec{k} ; \vec{K}, \vec{K}^{\prime}\right)^{*} M_{t_{1} t_{2}}\left(\vec{k} ; \vec{K}, \vec{K}^{\prime}\right)}{\omega_{t_{1}}(\vec{K})+\omega_{t_{2}}\left(\vec{K}^{\prime}\right)}
$$


where $\vec{K}^{\prime}$ is a dependent variable, given by $\vec{K}^{\prime}=(\vec{k}-\vec{K})_{\bmod 2 \pi / \ell}$; i.e., like $\vec{K}, \vec{K}^{\prime}$ also lies within the Brillouin zone, but with the difference $\left[\vec{K}^{\prime}-(\vec{k}-\right.$ $\vec{K})]_{a}=(2 \pi / \ell) \times$ integer. Here, as before, the subscript $a=x, y$ and $z$. We note that as $\vec{k} \rightarrow 0$, the limit $h(\vec{K}, \vec{k})$ is well behaved, on account of (5.74)(5.75). However, after the $\vec{K}$ integration, the function $I I(\vec{k})$ diverges as $\vec{k} \rightarrow 0$.

As in (5.57), write

$$
I I(\vec{k})=I I_{c}(\vec{k})+I I^{\prime}(\vec{k})
$$

with

$$
I I_{c}(\vec{k})=\int_{B} \frac{d^{3} K}{(2 \pi)^{3}} h_{c}(\vec{K}, \vec{k})
$$

and

$$
I I^{\prime}(\vec{k})=\int_{B} \frac{d^{3} K}{(2 \pi)^{3}}\left[h(\vec{K}, \vec{k})-h_{c}(\vec{K}, \vec{k})\right]
$$

Choose $h_{c}(\vec{K}, \vec{k})$ to be the integrand of $I I(\vec{k})$ without the $0^{t h}$-band approximation, i.e.

$$
\begin{aligned}
h_{c}(\vec{K}, \vec{k})=\frac{1}{4 k^{2}} & \frac{(K-|\vec{k}-\vec{K}|)^{2}}{K|\vec{k}-\vec{K}|(K+|\vec{k}-\vec{K}|)} \\
& \times\left(\delta_{a b}-\frac{K_{a} K_{b}}{K^{2}}\right)\left(\delta_{a b}-\frac{(\vec{k}-\vec{K})_{a}(\vec{k}-\vec{K})_{b}}{(\vec{k}-\vec{K})^{2}}\right),
\end{aligned}
$$

with the constraint that $\vec{K}$ lie within the Brillouin zone imposed as a boundary condition on the integral (5.86). One can readily verify that $I I^{\prime}(\vec{k})$ is free from divergence and obtain (see Appendix $\mathrm{C}$ for details)

$$
I I(\vec{k})=\frac{n}{24 \pi^{2}} g_{\ell}^{4}\left(\ln \frac{1}{k \ell}+\lambda^{(i i)}\right)
$$

where the constant

$$
\lambda^{(i i)}=\frac{23}{3}-11 \ln 2+\lambda_{c}+\lambda^{\prime \prime}
$$

with $\lambda_{c}$ given by (5.60) and

$$
\lambda^{\prime \prime}=24 \pi^{2} I I^{\prime}(0)=\int_{B} \frac{d^{3} \vec{K}}{(2 \pi)^{3}} \lim _{k \rightarrow 0}\left[h(\vec{K}, \vec{k})-h_{c}(\vec{K}, \vec{k})\right] .
$$


Note that in the limit when the magnitude $k \rightarrow 0$, the integrand remains dependent on the direction $\hat{k}=\vec{k} / k$. (See (C.17), (C.25) and (C.28) below.) In Appendix C, we show that $\lambda^{\prime \prime}=0.1238$ and therefore

$$
\lambda^{(i i)}=1.8362 .
$$

Substituting (5.88) into (5.81) and carrying out the integration over $\vec{k}$, we find

$$
\mathcal{E}^{(i i)}=-\frac{n}{24 \pi^{2}}\left(\ln \frac{R}{\ell}+\gamma+\lambda^{(i i)}\right) .
$$

Combining (5.65)-(5.66) and (5.91)-(5.92), we establish the constant $\lambda$ in (5.21) to be the one given by $(5.25)$; i.e.,

$$
\lambda=\lambda^{(i)}+\lambda^{(i i)}=3.3559 .
$$




\section{APPENDIX A}

In the following we wish to compare the lattice formulation given in this paper to the conventional ones in which the lattice structure consists of only discrete sites connected by links, but without being embedded within a continuum. An example of such a comparison for the case of a scalar field in one dimension was given at the end of Subsection 2.3. In this appendix we extend the comparison to the gauge theory.

Let it be agreed that the aim is to discretize only the spatial coordinates while time remains continuous. This violates relativistic symmetries, but no fixed spatial lattice can be invariant under the full Lorentz group anyway.

Take a simple cubic lattice of spacing $\ell$ and period $N \ell$ in each orthogonal direction; the volume associated with the full period is $\Omega=(N \ell)^{3}$. Conventionally, one labels the sites of the lattice as $j=\left(j_{1}, j_{2}, j_{3}\right)$ and attaches to each link a variable analogous to the vector potential, say $a_{j}, b_{j}, c_{j}$ on the links leaving site $j$ in the $x-, y-, z$-directions (as in (3.12)).

Consider first an Abelian gauge theory. The conventional lattice approach would be to write a lattice Lagrangian for a discrete system

$$
\begin{aligned}
\mathcal{L}_{d} & =\sum_{j} \frac{\ell^{3}}{2}\left[\left(\dot{a}_{j}-d_{j^{\prime}}+d_{j}\right)^{2}+\left(\dot{b}_{j}-d_{j^{\prime \prime}}+d_{j}\right)^{2}+\left(\dot{c}_{j}-d_{j^{\prime \prime \prime}}+d_{j}\right)^{2}\right] \\
& -\sum_{j} \frac{\ell}{2}\left[\left(a_{j}+b_{j^{\prime}}-a_{j^{\prime \prime}}-b_{j}\right)^{2}+\left(b_{j}+c_{j^{\prime \prime}}-b_{j^{\prime \prime \prime}}-c_{j}\right)^{2}+\left(c_{j}+a_{j^{\prime \prime \prime}}-c_{j^{\prime}}+a_{j}\right)^{2}\right]
\end{aligned}
$$

where, as in (3.18),

$$
\begin{aligned}
j^{\prime} & =\left(j_{1}+1, j_{2}, j_{3}\right), \\
j^{\prime \prime} & =\left(j_{1}, j_{2}+1, j_{3}\right), \\
j^{\prime \prime \prime} & =\left(j_{1}, j_{2}, j_{3}+1\right) ;
\end{aligned}
$$

the first term of (A.1) is "electric" and the second "magnetic". The new variable $d_{j}$ attached to site $j$ is analogous to the "time" component of the vector potential.

One notes that $\mathcal{L}_{d}$ is invariant under the lattice gauge transformation 


$$
\begin{aligned}
a_{j} & \rightarrow a_{j}+\lambda_{j^{\prime}}-\lambda_{j}, \\
b_{j} & \rightarrow b_{j}+\lambda_{j^{\prime \prime}}-\lambda_{j}, \\
c_{j} & \rightarrow c_{j}+\lambda_{j^{\prime \prime \prime}}-\lambda_{j}, \\
d_{j} & \rightarrow d_{j}+\dot{\lambda}_{j}
\end{aligned}
$$

where $\lambda_{j}$ is an arbitrary quantity attached to site $j$. One may take advantage of this to pass either to the lattice time-axial gauge where

$$
d_{j}=0 \quad(\text { all } j)
$$

or to the lattice Coulomb gauge where

$$
a_{j}-a_{j_{-}^{\prime}}+b_{j}-b_{j_{-}^{\prime \prime}}+c_{j}-c_{j_{-}^{\prime \prime \prime}}=0
$$

here, as in (4.16),

$$
\begin{aligned}
& j_{-}^{\prime}=\left(j_{1}-1, j_{2}, j_{3}\right), \\
& j_{-}^{\prime \prime}=\left(j_{1}, j_{2}-1, j_{3}\right), \\
& j_{-}^{\prime \prime \prime}=\left(j_{1}+1, j_{2}, j_{3}-1\right) .
\end{aligned}
$$

In time-axial gauge one may write the Lagrangian (A.1) in "momentum space":

$$
\mathcal{L}_{d}=\sum_{\vec{K}}\left(\dot{q}^{\dagger} \dot{q}-q^{\dagger}\left(u^{2}-u \tilde{u}\right) q\right)
$$

where $q=q(\vec{K})$ and $u=u(\vec{K})$ are $3 \times 1$ column matrices. The components of $q$ are the Fourier coefficients of $a_{j}, b_{j}, c_{j}$. Those of $u$ are

$$
u_{a}(\vec{\theta})=\frac{2}{\ell} \sin \frac{\theta_{a}}{2}
$$

with $\vec{\theta}=\vec{K} \ell$. Evidently there are redundant degrees of freedom, which one may eliminate by writing

$$
Q_{\ell}(\vec{K})=\hat{u}(\vec{K}) \cdot \vec{q}(\vec{K})
$$

where $(\hat{u})_{a}=u_{a} / \sqrt{u^{2}}$, and

$$
Q_{t}(\vec{K})=\hat{\epsilon}_{t}(\vec{K}) \cdot \vec{q}(\vec{K}) \quad(t=1,2)
$$


with $\hat{u}, \hat{\epsilon}_{1}, \hat{\epsilon}_{2}$ forming a right-handed orthonormal system. Then (A.7) becomes

$$
\mathcal{L}_{d}=\sum_{\vec{K}}\left(\dot{Q}_{\ell}^{\dagger} \dot{Q}_{\ell}+\sum_{t=1}^{2}\left(\dot{Q}_{t}^{\dagger} \dot{Q}_{t}-u^{2} Q_{t}^{\dagger} Q_{t}\right)\right)
$$

In this gauge there is a supplementary condition on the state vector,

$$
\frac{\partial}{\partial Q_{\ell}(\vec{K})}<Q \mid>=0
$$

in the absence of sources, derived from the lattice analog of Gauss' law. Hence the variables $Q_{\ell}$ can be ignored and the Hamiltonian written as

$$
\mathcal{H}_{d}=\sum_{\vec{K}} \sum_{t=1}^{2}\left(P_{t}^{\dagger} P_{t}+u^{2} Q_{t}^{\dagger} Q_{t}\right)
$$

where $P_{t}(\vec{K})$ is the conjugate momentum of $Q_{t}(\vec{K})$.

Alternatively, in lattice Coulomb gauge one has from the outset $Q_{\ell}=0$, all $\vec{K} \neq 0$, and the variables $d_{j}$ are decoupled from $Q_{t}$. In the absence of sources the $d_{j}$ vanish and the Hamiltonian again takes the form (A.13).

All this proceeds smoothly, but one encounters difficulties in extending it to a non-Abelian theory. For example, how is one to represent the nonlinear term in the magnetic field?

Two different approaches are possible. The first is to go over to a compact formulation ${ }^{2}$ based not on $a_{j}, b_{j}, c_{j}$ but on $e^{i \ell a_{j}}, e^{i \ell b_{j}}, e^{i \ell c_{j}}$. The disadvantage is that this formulation, for nonzero $\ell$, is no longer connected with the true continuum theory based on

$$
\mathcal{L}=-\int d^{3} r \frac{1}{2}\left(\frac{\partial A_{\nu}}{\partial x_{\mu}}-\frac{\partial A_{\mu}}{\partial x_{\nu}}\right)^{2}
$$

where the $A_{\mu}$ are continuous fields with gauge transformation

$$
A_{\mu} \rightarrow A_{\mu}+\frac{\partial \Lambda}{\partial x_{\mu}}
$$

The "compact" theory derived by nonlinearizing (A.1) and (A.3) is an abstract model of the original theory, not a concrete approximation to it.

The second approach, which we pursue in this paper, is to seek a noncompact lattice theory that can serve as a first approximation in a systematic way 
which can approach the exact continuum theory, without requiring $\ell$ to approach zero. Thus (A.14) and (A.15) will be our starting point, not (A.1) and (A.3); the lattice variables $a_{j}, b_{j}, c_{j}$ will enter only as parameters controlling the few degrees of freedom allowed to $\vec{A}$ in the zeroth approximation.

A natural way to implement this idea is to make each component of $\vec{A}$ a constant along any link in the corresponding direction, and interpolate linearly between links. Referring to the lump functions defined above, of which $L_{1}$ is a constant and $L_{2}$ is linear, we see that this amounts to setting

$$
\begin{aligned}
\vec{A}(\vec{r})= & \sum_{j}\left[a_{j} \hat{x} L_{1}\left(x-j_{1} \ell\right) L_{2}\left(y-j_{2} \ell\right) L_{2}\left(z-j_{3} \ell\right)\right. \\
& +b_{j} \hat{y} L_{2}\left(x-j_{1} \ell\right) L_{1}\left(y-j_{2} \ell\right) L_{2}\left(z-j_{3} \ell\right) \\
& \left.+c_{j} \hat{z} L_{2}\left(x-j_{1} \ell\right) L_{2}\left(y-j_{2} \ell\right) L_{1}\left(z-j_{3} \ell\right)\right] .
\end{aligned}
$$

The idea is to substitute this expression into (A.14) so as to obtain a Lagrangian depending on the "effective" lattice variables $a_{j}, b_{j}, c_{j}$. (Writing $\vec{A}(\vec{r}), L_{1}(x)$ and $L_{2}(x)$ as $\vec{V}(\vec{r}), C(x)$ and $\Delta(x)$, we see that (A.16) is the same as (3.7)).

For an Abelian theory this idea works nicely in time-axial gauge because the time-independent gauge transformation (A.3) actually generates a transformation of the form (A.15) on the continuous fields defined in (A.16). As shown in (3.22), this makes it possible to implement the subsidiary state condition derived from the continuum Gauss' law by eliminating some of the lattice variables similar to (A.13). But this no longer works in a non-Abelian theory: the non-Abelian version of (A.1) is not invariant under any transformation of the type (A.3).

For the non-Abelian theory, we propose instead to work in Coulomb gauge (or Coulomb-like gauge (3.84)). We find that if we replace the function $L_{1}$ in (A.16) by $L_{3}$, then on account of (2.3) the condition (A.5) on the lattice variables is sufficient to ensure the true Coulomb gauge condition

$$
\vec{\nabla} \cdot \vec{A}=0
$$

at every point in space. Moreover, if the higher band functions are built in the manner indicated by (3.73), then (A.17) can be satisfied independently within each band at every point in space. This remains true in non-Abelian theory because the Coulomb gauge condition is linear. The details are carried out in 
Subsection 3.2 and Section 4. Identical considerations can be applied to any gauge in which the gauge-fixing condition has no nonlinear terms, as in (3.84). 


\section{APPENDIX B}

In this appendix, we give another method for the removal of redundant lattice coordinate space variables, alternative to the one given in Section 4.3.

The objective is to eliminate the $\mathcal{N}+2$ redundant variables among the set

$$
\left\{\xi_{j}^{\ell}, \eta_{j}^{\ell}, \zeta_{j}^{\ell}\right\}
$$

of $3 \mathcal{N}$ plaquette flux variables. Again, the group superscript $\ell$ will be kept fixed, and the factor of its $n^{2}-1$ degrees of freedom due to $S U(n)$ will not be included.

Theorem. For any plaquette flux distribution (B.1), through (4.29) in which

$$
\left\{\xi_{j}^{\ell}, \eta_{j}^{\ell}, \zeta_{j}^{\ell}\right\} \rightarrow\left\{\xi_{j}^{\prime \ell}, \eta_{j}^{\prime \ell}, \zeta_{j}^{\prime \ell}\right\}
$$

the transformed plaquette fluxes can be made to satisfy the lattice divergencefree equation:

$$
\xi_{j^{\prime}}^{\prime \ell}-\xi_{j}^{\prime \ell}+\eta_{j^{\prime \prime}}^{\prime \ell}-\eta_{j}^{\prime \ell}+\zeta_{j^{\prime \prime \prime}}^{\prime \ell}-\zeta_{j}^{\prime \ell}=0
$$

at all $j$, with the sites $j^{\prime}, j^{\prime \prime}$ and $j^{\prime \prime \prime}$ given by (3.18).

Proof. Consider the cubic cell $\tau_{j}$ that has $X_{j}, Y_{j}$ and $Z_{j}$ of Figure 3(a) as three of its six surfaces. The other three surfaces are (in the same notation) $X_{j^{\prime}}, Y_{j^{\prime \prime}}$ and $Z_{j^{\prime \prime \prime}}$. Define the lattice differences

$$
\begin{aligned}
& \partial_{x}\left(I_{x}^{\ell}\right)_{j} \equiv \xi_{j^{\prime}}^{\ell}-\xi_{j}^{\ell}, \\
& \partial_{y}\left(I_{y}^{\ell}\right)_{j} \equiv \eta_{j^{\prime \prime}}^{\ell}-\eta_{j}^{\ell},
\end{aligned}
$$

and

$$
\partial_{z}\left(I_{z}^{\ell}\right)_{j} \equiv \zeta_{j^{\prime \prime \prime}}^{\ell}-\zeta_{j}^{\ell}
$$

to be the net flux flowing out from $\tau_{j}$ along the $x, y$ and $z$ axes. The lattice divergence

$$
\vec{\partial} \cdot \vec{I}_{j}^{\ell} \equiv \sum_{a=x}^{z} \partial_{a}\left(I_{a}^{\ell}\right)_{j}
$$


represents the total flux leaving $\tau_{j}$.

Next, we turn to the dual lattice $L_{D}$ : The center of $\tau_{j}$ in the original lattice $L$ becomes the lattice site $j$ in $L_{D}$. Correspondingly, $\xi_{j}^{\ell}$, $\eta_{j}^{\ell}$ and $\zeta_{j}^{\ell}$ denote link currents, and $\vec{\partial} \cdot \vec{I}_{j}{ }^{\ell}$ is the net current leaving the site $j$ (with the components of $\vec{I}_{j}^{\ell}$ now defined on the links $\overline{j^{\prime} j}, \overline{j^{\prime \prime} j}$ and $\overline{j^{\prime \prime \prime} j}$ in $L_{D}$ ). Likewise, introduce

$$
\begin{aligned}
\partial_{x}^{2} \chi_{j}^{\ell} & \equiv \chi_{j^{\prime}}^{\ell}-2 \chi_{j}^{\ell}+\chi_{j_{-}^{\prime}}^{\ell}, \\
\partial_{y}^{2} \chi_{j}^{\ell} & \equiv \chi_{j^{\prime \prime}}^{\ell}-2 \chi_{j}^{\ell}+\chi_{j_{-}^{\ell \prime}}^{\ell}, \\
\partial_{z}^{2} \chi_{j}^{\ell} & \equiv \chi_{j^{\prime \prime \prime}}^{\ell}-2 \chi_{j}^{\ell}+\chi_{j_{-}^{\prime \prime \prime}}^{\ell}
\end{aligned}
$$

and

$$
\partial^{2} \chi_{j}^{\ell} \equiv \sum_{a=x}^{z} \partial_{a}^{2} \chi_{j}^{\ell}
$$

to be the second-order lattice differences and the lattice Laplacian. The sites $j, j^{\prime}, \cdots j_{-}^{\prime \prime \prime}$ in $L_{D}$ are dual to the cubic cells $\tau_{j}, \tau_{j^{\prime}}, \cdots \tau_{j_{-}^{\prime \prime \prime}}$ in $L$ which, in turn, are related to the sites $j, j^{\prime}, \cdots j_{-}^{\prime \prime \prime}$ in $L$, given by (3.18) and (4.16), in the way described above.

Equation (B.3) states that through the transformation (4.29), $\vec{I}_{j} \ell$ becomes $\vec{I}_{j}^{\prime \ell}$ which satisfies $\vec{\partial} \cdot \vec{I}_{j}^{\prime \ell}=0$; therefore

$$
-\partial^{2} \chi_{j}^{\ell}=\vec{\partial} \cdot \vec{I}_{j}^{\ell} \equiv \rho_{j}^{\ell}
$$

We can readily obtain the solution through the lattice Green's function

$$
\mathcal{G}(\vec{r}) \equiv \sum_{\vec{K} \neq 0} \frac{1}{2 \mathcal{N}}\left[3-\sum_{a} \cos \theta_{a}\right]^{-1} e^{i \vec{K} \cdot \vec{r}}
$$

where $\theta_{a}=K_{a} \ell$ as before, and the sum extends over the Brillouin zone but with its origin excluded. The result is

$$
\chi_{j}^{\ell}=\sum_{i} \mathcal{G}\left(\vec{r}_{j}-\vec{r}_{i}\right) \rho_{i}^{\ell}
$$

The theorem is then established. 
The function $\mathcal{G}(\vec{r})$ has been well studied in the literature. For example, at $\vec{r}=0$ and $\mathcal{N} \rightarrow \infty$

$$
\mathcal{G}(0)=0.25273
$$

which can also be expressed ${ }^{10}$ in terms of the elliptic integral

$$
\mathcal{G}(0)=\frac{2}{\pi^{2}}(18+12 \sqrt{2}-10 \sqrt{3}-7 \sqrt{6}) \times K^{2}[(2-\sqrt{3})(3-\sqrt{2})]
$$

where

$$
K(k)=\int_{0}^{\frac{\pi}{2}}\left(1-k^{2} \sin ^{2} \phi\right)^{-\frac{1}{2}} d \phi
$$

Returning to the problem of eliminating redundant variables, we note that (B.3) imposes $\mathcal{N}-1$ conditions. Since any plaquette flux distribution can be transformed into one that satisfies (B.3), the resulting divergence-free distribution consists only of

$$
3 \mathcal{N}-(\mathcal{N}-1)=2 \mathcal{N}+1
$$

independent plaquette-flux variables. As explained at the end of Section 4.3, we can arbitrarily set three surface plaquette fluxes of $L$ to zero (one along each Cartesian direction) without affecting the link-flux distribution $\left(a_{j}^{\ell}, b_{j}^{\ell}, c_{j}^{\ell}\right)$. This and (B.14) complete the reduction process: the number of independent variables in $\left(\xi_{j}^{\ell}, \eta_{j}^{\ell}, \zeta_{j}^{\ell}\right)$ becomes $2 \mathcal{N}+1-3=2 \mathcal{N}-2$, in accordance with (4.30). 


\section{APPENDIX C}

\section{C.1. The Integral $I_{c}(\vec{k})$}

To calculate the integral $I_{c}(\vec{k})$ given by $(5.58)$ for $k \ell<<1$, we divide the Brillouin zone, $-\frac{\pi}{\ell} \leq K_{a} \leq \frac{\pi}{\ell}$ (where $a=x, y, z$ ), into two regions: the interior of a small sphere $s$ with its center at the origin $\vec{K}=0$ and the volume outside the sphere. The radius of the sphere, $K_{0}$, is chosen such that $k<<K_{0}<<\frac{\pi}{\ell}$. Correspondingly, the integral (5.58) can be separated into two terms:

$$
I_{c}(k)=I_{\mathrm{in}}+I_{\mathrm{out}}
$$

where

$$
I_{\text {in }}=\int_{\text {in }} \frac{d^{3} K}{(2 \pi)^{3}} f_{c}(\vec{K}, \vec{k})
$$

and

$$
I_{\text {out }}=\int_{\text {out }} \frac{d^{3} K}{(2 \pi)^{3}} f_{c}(\vec{K}, \vec{k})
$$

with $f_{c}(\vec{K}, \vec{k})$ given by (5.55), the integral (C.2) referring to the integration inside the sphere $s$ and (C.3) for the integration outside $s$ but within the Brillouin zone. Since $I_{\text {in }}$ contains $\ln k \ell, k$ may not be set to zero in its integrand. A straightforward calculation in spherical coordinates yields:

$$
I_{\text {in }}=\frac{1}{6 \pi^{2}}\left(\ln \frac{K_{0}}{k}+\frac{1}{3}\right)
$$

where we have dropped the terms that vanish in the limit $k \rightarrow 0$. To the same order, $k$ may be set to zero in the integrand of $I_{\text {out }}$, on account of the low momentum cutoff $\left(K>K_{0}\right)$. We have

$$
I_{\text {out }}=\int_{\text {out }} \frac{d^{3} K}{(2 \pi)^{3}} \lim _{k \rightarrow 0} f_{c}(\vec{K}, \vec{k}) .
$$

It follows from (5.55) and (5.56) that

$$
\lim _{k \rightarrow 0} f_{c}(\vec{K}, \vec{k})=\frac{1}{2 K^{3}}\left[1-\frac{(\hat{k} \cdot \vec{K})^{2}}{K^{2}}\right] .
$$

Because of cubic symmetry, we may replace $(\hat{k} \cdot \vec{K})^{2} / K^{2}$ by $\frac{1}{3}$ in the integration; (C.5) becomes 


$$
I_{\text {out }}=\frac{1}{3} \int_{\text {out }} \frac{d^{3} \kappa}{(2 \pi)^{3}} \frac{1}{\kappa^{3}}
$$

in which we introduce the dimensionless vector $\vec{\kappa} \equiv \frac{\ell}{\pi} \vec{K}$, whose Cartesian coordinates will be denoted by $(x, y, z)$, so that its magnitude is $\kappa=\sqrt{x^{2}+y^{2}+z^{2}}$. The integration domain of (C.7) in terms of $\vec{\kappa}$ is given by $-1 \leq x, y, z \leq 1$ and $\kappa>\frac{\ell}{\pi} K_{0}$. In terms of spherical coordinates with $a$ and $b$ denoting the polar and azimuthal angles, we obtain

$$
I_{\mathrm{out}}=\frac{1}{24 \pi^{3}} \int_{0}^{2 \pi} d b \int_{0}^{\pi} d a \sin a \ln \kappa(a, b)-\frac{1}{6 \pi^{2}} \ln \frac{K_{0} \ell}{\pi}
$$

where $\kappa(a, b)$ is the radial coordinate of a point on the boundary of the Brillouin zone in the direction specified by the angles $a$ and $b$. The Brillouin zone is a cube bounded by six faces: $x= \pm 1, y= \pm 1$ and $z= \pm 1$. Because of cubic symmetry, we need only integrate over one of these faces and then multiply the result by six. Take the surface at $z=1$ : we have

$$
\begin{aligned}
& x=\tan a \cos b, \\
& y=\tan a \sin b,
\end{aligned}
$$

and

$$
\kappa(a, b)=\sec a
$$

(C.8) reduces to

$$
I_{\mathrm{out}}=\frac{1}{8 \pi^{3}} \int_{-1}^{1} d x \int_{-1}^{1} d y \frac{\ln \left(x^{2}+y^{2}+1\right)}{\left(x^{2}+y^{2}+1\right)^{\frac{3}{2}}}+\frac{1}{6 \pi^{2}} \ln \frac{\pi}{K_{0} \ell}
$$

Carrying out the integration over $y$, we find

$$
I_{\mathrm{out}}=\frac{1}{6 \pi^{2}}\left(\ln \frac{\pi}{K_{0} \ell}+1-\frac{6}{\pi} \int_{0}^{1} d x \frac{\ln \left(x^{2}+2\right)}{\left(x^{2}+1\right) \sqrt{x^{2}+2}}\right) .
$$

Combining (C.4) and (C.12), we establish (5.60) and (5.61). 


\section{C.2. The Integral $I I_{c}(\vec{k})$}

Following the same method as in the above section, we separate the integral (5.85) into two terms,

$$
I I_{c}(k)=I I_{\mathrm{in}}+I I_{\mathrm{out}}
$$

where, as in (C.1)-(C.3),

$$
I I_{\text {in }}=\int_{\text {in }} \frac{d^{3} K}{(2 \pi)^{3}} h_{c}(\vec{K}, \vec{k})
$$

is the integration inside the same small sphere $s$ and

$$
I I_{\text {out }}=\int_{\text {out }} \frac{d^{3} K}{(2 \pi)^{3}} h_{c}(\vec{K}, \vec{k})
$$

is the integration outside $s$ but inside the Brillouin zone. The integrand $h_{c}(\vec{K}, \vec{k})$ is given by (5.87). In the limit $k \rightarrow 0$ we have

$$
I I_{\text {out }}=\int_{\text {out }} \frac{d^{3} K}{(2 \pi)^{3}} \lim _{k \rightarrow 0} h_{c}(\vec{K}, \vec{k})=\frac{1}{4} I_{\text {out }}
$$

since, on account of (5.87),

$$
\lim _{k \rightarrow 0} h_{c}(\vec{K}, \vec{k})=\frac{1}{4} \frac{(\hat{k} \cdot \vec{K})^{2}}{K^{5}}
$$

which can be replaced by $\left(12 K^{3}\right)^{-1}$ because of cubic symmetry. Therefore

$$
I I_{\mathrm{out}}=\frac{1}{24 \pi^{2}}\left(\ln \frac{\pi}{K_{0} \ell}+1-\frac{6}{\pi} \int_{0}^{1} d x \frac{\ln \left(x^{2}+2\right)}{\left(x^{2}+1\right) \sqrt{x^{2}+2}}\right)
$$

where, as before, $K_{0}$ is the radius of the small sphere $s$.

To carry out the integration inside $s$ for $I I_{\text {in }}$, we find it convenient to introduce the following prolate spheroidal coordinates for the Bloch wave number vector $\vec{K}$ :

$$
\begin{aligned}
& K_{x}=\frac{k}{2} \sqrt{\left(\xi^{2}-1\right)\left(1-\eta^{2}\right)} \cos \phi, \\
& K_{y}=\frac{k}{2} \sqrt{\left(\xi^{2}-1\right)\left(1-\eta^{2}\right)} \sin \phi,
\end{aligned}
$$




$$
K_{z}=\frac{k}{2}(\xi \eta+1)
$$

with $0 \leq \phi \leq 2 \pi,-1 \leq \eta \leq 1$ and $\xi \leq 1$. To derive the $\ln K_{0} / k$ term and the constant term of $I I_{\text {in }}$ in the limit $k \rightarrow 0$, we may approximate the spherical domain of the integration by a prolate spheroid with foci $(0,0,0)$ and $(0,0, k)$ and with the length of its semi-major axis equal to $K_{0}$, which corresponds to the upper limit of $\xi<\frac{2 K_{0}}{k}$ in the $\xi$-integration. The error due to the approximation vanishes in the limit $k \rightarrow 0$. We find

$$
\begin{aligned}
I I_{\mathrm{in}} & =\frac{1}{32 \pi^{2}} \int_{1}^{2 K_{0} / k} d \xi \int_{-1}^{1} d \eta \frac{\eta^{2}}{\xi}\left[1+\frac{\left(\xi^{2}+\eta^{2}-2\right)^{2}}{\left(\xi^{2}-\eta^{2}\right)^{2}}\right] \\
& =\frac{1}{24 \pi^{2}}\left(\ln \frac{K_{0}}{k}+8-11 \ln 2\right) .
\end{aligned}
$$

Combining (C.18) and (C.20), we derive the expression of $I I_{c}(k)$ when $k \ell \rightarrow 0$; this leads to (5.88) and (5.89), except for the constant $\lambda^{\prime \prime}$ which will be evaluated below.

\section{C.3. The constants $\lambda^{\prime}$ and $\lambda^{\prime \prime}$}

The expression for the constant $\lambda^{\prime}$ follows from (5.53), (5.54), (5.55), (5.59) and (5.62) and it reads:

$$
\lambda^{\prime}=2 \pi^{2} \int_{B} \frac{d^{3} K}{(2 \pi)^{3}}\left[\sum_{a, t} \frac{\hat{\epsilon}_{t}(\vec{K})_{a} \hat{\epsilon}_{t}(\vec{K})_{a}}{2 \omega_{t}(\vec{K})} \sum_{\vec{m}} \frac{\Omega C_{a}(\vec{\theta} \mid \vec{m})^{2}}{\left(\vec{K}+\frac{2 \pi \vec{m}}{\ell}\right)^{2}}-\frac{1}{K^{3}}\right]
$$

To derive the expression for $\lambda^{\prime \prime}$, we separate the summation $\sum_{t_{1} t_{2}}$ in $h(\vec{K}, \vec{k})$ of $(5.83)$ into a sum over the same polarization, $t_{1}=t_{2}=t$, and another sum over different polarizations $t_{1} \neq t_{2}$, i.e.

$$
h(\vec{K}, \vec{k})=h_{1}(\vec{K}, \vec{k})+h_{2}(\vec{K}, \vec{k})
$$

where

$$
h_{1}(\vec{K}, \vec{k})=\frac{1}{k^{2}} \sum_{t} \frac{M_{t t}\left(\vec{k} ; \vec{K}, \vec{K}^{\prime}\right)^{*} M_{t t}\left(\vec{k} ; \vec{K}, \vec{K}^{\prime}\right)}{\omega_{t}(\vec{K})+\omega_{t}\left(\vec{K}^{\prime}\right)}
$$


and

$$
h_{2}(\vec{K}, \vec{k})=\frac{1}{k^{2}} \sum_{t_{1} \neq t_{2}} \frac{M_{t_{1} t_{2}}\left(\vec{k} ; \vec{K}, \vec{K}^{\prime}\right)^{*} M_{t_{1} t_{2}}\left(\vec{k} ; \vec{K}, \vec{K}^{\prime}\right)}{\omega_{t_{1}}(\vec{K})+\omega_{t_{2}}\left(\vec{K}^{\prime}\right)}
$$

with $\vec{K}^{\prime}$ defined by the prescription following (5.83). The limit $k \rightarrow 0$ of $h_{1}(\vec{K}, k)$ can be readily obtained, and we have

$$
\lim _{k \rightarrow 0} h_{1}(\vec{K}, \vec{k})=\frac{1}{4} \sum_{t} \frac{\left(\hat{k} \cdot \vec{\nabla}_{\vec{K}} \omega_{t}\right)^{2}}{\omega_{t}^{3}(\vec{K})} .
$$

In the same limit, using the Fourier expansion (3.66) and (3.67), we find

$$
\int d^{3} r F_{a}(\vec{K} \mid \vec{r})^{*} F_{a}\left(\vec{K}^{\prime} \mid \vec{r}\right)^{*} e^{i \vec{k} \cdot \vec{r}}=N^{3} \sum_{\vec{m}} \mathcal{C}_{a}(\vec{\theta} \mid \vec{m}) \mathcal{C}_{a}(\vec{\theta}-\vec{k} \ell \mid \vec{m})=1+O\left(k^{2} \ell^{2}\right)
$$

and therefore

$$
M_{t_{1} t_{2}}\left(\vec{k} ; \vec{K}, \vec{K}^{\prime}\right)=\frac{i\left[\omega_{t_{1}}(\vec{K})-\omega_{t_{2}}(\vec{K})\right]}{2 \sqrt{\omega_{t_{1}}(\vec{K}) \omega_{t_{2}}(\vec{K})}} \sum_{a} \hat{\epsilon}_{t_{1}}(\vec{K})_{a} \vec{k} \cdot \vec{\nabla}_{\vec{K}} \hat{\epsilon}_{t_{2}}(-\vec{K})_{a}+O\left(k^{2} \ell^{2}\right)
$$

for $t_{1} \neq t_{2}$, in accordance with (5.75). Substituting (C.27) into (C.24) we obtain

$$
\begin{aligned}
\lim _{k \rightarrow 0} h_{2}(\vec{K}, \vec{k})= & \frac{1}{4} \sum_{t_{1} \neq t_{2}} \frac{\left[\omega_{t_{1}}(\vec{K})-\omega_{t_{2}}(\vec{K})\right]^{2}}{\omega_{t_{1}}(\vec{K}) \omega_{t_{2}}(\vec{K})\left[\omega_{t_{1}}(\vec{K})+\omega_{t_{2}}(\vec{K})\right]} \\
& \times \sum_{a, b}\left[\hat{\epsilon}_{t_{1}}(\vec{K})_{a} \hat{k} \cdot \vec{\nabla}_{\vec{K}} \hat{\epsilon}_{t_{2}}(-\vec{K})_{a}\right]\left[\hat{\epsilon}_{t_{1}}(\vec{K})_{b} \hat{k} \cdot \vec{\nabla}_{\vec{K}} \hat{\epsilon}_{t_{2}}(-\vec{K})_{b}\right] .
\end{aligned}
$$

Following the definitions (5.90) and (5.86) we have

$$
\lambda^{\prime \prime}=\lambda_{1}^{\prime \prime}+\lambda_{2}^{\prime \prime}
$$

where

$$
\begin{aligned}
\lambda_{1}^{\prime \prime} & =24 \pi^{2} \int \frac{d^{3} K}{(2 \pi)^{3}} \lim _{k \rightarrow 0}\left[h_{1}(\vec{K}, \vec{k})-h_{c}(\vec{K}, \vec{k})\right] \\
& =2 \pi^{2} \int_{B} \frac{d^{3} K}{(2 \pi)^{3}}\left[\sum_{t} \frac{\left|\vec{\nabla}_{\vec{K}} \omega_{t}\right|^{2}}{2 \omega_{t}^{3}(\vec{K})}-\frac{1}{K^{3}}\right]
\end{aligned}
$$


and

$$
\begin{aligned}
\lambda_{2}^{\prime \prime} & =2 \pi^{2} \int \frac{d^{3} K}{(2 \pi)^{3}} \sum_{t_{1} \neq t_{2}} \frac{\left[\omega_{t_{1}}(\vec{K})-\omega_{t_{2}}(\vec{K})\right]^{2}}{\omega_{t_{1}}(\vec{K}) \omega_{t_{2}}(\vec{K})\left[\omega_{t_{1}}(\vec{K})+\omega_{t_{2}}(\vec{K})\right]} \\
& \times \sum_{a, b, c} \hat{\epsilon}_{t_{1}}(\vec{K})_{a} \frac{\partial \hat{\epsilon}_{t_{2}}(-\vec{K})_{a}}{\partial K_{c}} \hat{\epsilon}_{t_{1}}(\vec{K})_{b} \frac{\partial \hat{\epsilon}_{t_{2}}(-\vec{K})_{b}}{\partial K_{c}}
\end{aligned}
$$

Both integrations (C.30) and (C.31) are well defined. The result is $\lambda^{\prime \prime}=0.1238$.

\section{C.4. The Remainder $\delta$}

The function $\delta\left(\ell, \vec{R}_{1}, \vec{R}_{2}\right)$ is defined by (5.21)-(5.23), which relates $g_{R}^{2}$ to $g_{\ell}^{2}$. From (5.16)-(5.17), we see that $g_{R}^{2}$ appears as the coefficient of $e_{1}^{\ell} e_{2}^{\ell} / 4 \pi R$ in $E_{12}$; the latter can in turn be written as (neglecting $O\left(g_{\ell}^{6}\right)$ )

$$
E_{12}=g_{\ell}^{2} \frac{e_{1}^{\ell} e_{2}^{\ell}}{4 \pi R}+g_{\ell}^{4}\left(\mathcal{E}^{(i)}+\mathcal{E}^{(i i)}\right)
$$

It follows then

$$
\delta\left(\ell, \vec{R}_{1}, \vec{R}_{2}\right)=\frac{96 \pi^{3} R}{11 n e_{1}^{\ell} e_{2}^{\ell}}\left(\mathcal{E}^{(i)}+\mathcal{E}^{(i i)}\right)-\ln \frac{R}{\ell}-\gamma-\lambda
$$

where $\gamma$ is the Euler constant, $\lambda$ is given by (5.25),

$$
\mathcal{E}^{(i)}=3 n e_{1}^{\ell} e_{2}^{\ell} \int \frac{d^{3} k}{(2 \pi)^{3}} \frac{e^{i \vec{k} \cdot \vec{R}}}{k^{2}} I(\vec{k})
$$

with $I(\vec{k})$ given by $(5.52)$, and $\mathcal{E}^{(i i)}$ given by a similar expression (5.76). When $\ell \rightarrow 0, \delta$ satisfies

$$
\lim _{\ell \rightarrow 0} \delta\left(\ell, \vec{R}_{1}, \vec{R}_{2}\right)=0
$$

since that is the condition for the determination of $\lambda$. In general, the dependence of $\delta$ on $\ell, \vec{R}_{1}$ and $\vec{R}_{2}$ is not a simple one, though well defined through (C.33).

Because of (5.50) and (5.78), both $\mathcal{E}^{(i)}$ and $\mathcal{E}^{(i i)}$ are periodic in

$$
\vec{R}_{\mathrm{cm}}=\frac{1}{2}\left(\vec{R}_{1}+\vec{R}_{2}\right)
$$


with the periodicity of the lattice. It is convenient to average $\vec{R}_{\mathrm{cm}}$ over a unit lattice cell; this gives a $\langle\delta\rangle_{\text {av }}$ which depends only on $\ell$ and $\vec{R}=\vec{R}_{2}-\vec{R}_{1}$. For $\langle\delta\rangle_{\text {av }}$, we may set

$$
\vec{m}_{1}=\vec{m}_{2} \quad \text { and } \quad \vec{m}=\vec{m}^{\prime}
$$

in (5.52)-(5.53) and (5.76)-(5.77). Thus, the integrand in (C.34) can be written as

$$
I(\vec{k})=\sum_{\vec{m}} \int_{B} \frac{d^{3} K}{(2 \pi)^{3}} f_{\vec{m} \vec{m}}(\vec{K}, \vec{k})
$$

where

$$
f_{\vec{m} \vec{m}}(\vec{K}, \vec{k})=k^{-2} \Omega^{3} \sum_{t} \frac{\left[\sum_{a} \mathcal{C}_{a}(\vec{\theta} \mid \vec{m}) \hat{\epsilon}_{t}(\vec{K})_{a} k_{a}\right]^{2}}{2 \omega_{t}(\vec{K})\left(\vec{k}+\vec{K}-\frac{2 \pi \vec{m}}{\ell}\right)^{2}}
$$

Likewise, we have

$$
\mathcal{E}^{(i i)}=-n e_{1}^{\ell} e_{2}^{\ell} \int \frac{d^{3} k}{(2 \pi)^{3}} \frac{e^{i \vec{k} \cdot \vec{R}}}{k^{2}} I I(\vec{k})
$$

where

$$
I I(\vec{k})=\int_{B} \frac{d^{3} \vec{K}}{(2 \pi)^{3}} h(\vec{K}, \vec{k})
$$

and $h(\vec{K}, \vec{k})$ is given by (5.83). Substituting the above $\mathcal{E}^{(i)}$ and $\mathcal{E}^{(i i)}$ into (C.33), we derive the expression for $\langle\delta\rangle_{\mathrm{av}}$. 


\section{REFERENCES}

1. R. Friedberg, T. D. Lee and Y. Pang, J. Math. Phys. 35(11), 5600 (1994).

2. K. Wilson, Phys.Rev. D10, 2455 (1974); J. B. Kogut and L. Susskind, Phys.Rev. D11, 395 (1975). M. Creutz, Phys.Rev. D21, 2308 (1980).

3. Lattice gauge theories have been extensively studied in the literature. See Lattice 93, Nucl.Phys. (Proceedings Supplement) B34 (1994), eds. T. Draper, S. Gottlieb, A. Soni and D. Toussaint, for recent reviews of the subject, and the references cited therein. Noncompact lattice gauge theory has been discussed by K. Cahill, ibid., 231; A. Patrascioiu, E. Seiler and I. Stamatescu, Phys.Lett. B107, 364 (1981); I. Stamatescu, U. Wolff and D. Zwanziger, Nucl.Phys. B225 [FS9], 377 (1983); E. Seiler, I. Stamatescu and D. Zwanziger, Nucl.Phys. B239, 177 and 201 (1984); but their formulations are quite different from the one given in this paper.

4. T. D. Lee, Phys.Lett. B122, 217 (1983); R. Friedberg and T. D. Lee, Nucl.Phys. B225, 1 (1983).

5. See N. H. Christ and T. D. Lee, Phys.Rev. D22, 939 (1980), and the references cited therein.

6. T. D. Lee and C. N. Yang, Phys.Rev. 128, 885 (1962).

7. L. D. Faddeev and V. N. Popov, Phys.Lett. 25B, 29 (1967).

8. D. J. Gross and F. Wilczek, Phys.Rev.Lett. 30, 1343 (1973) and H. Politzer, ibid., 1346.

9. An uncommon feature here is the combined use of two different kinds of wave number vectors, with $\vec{K}$ related to the lattice structure, and $\vec{p}, \vec{p}^{\prime}, \vec{q}$ the usual continuum variables, as displayed explicitly in (5.43)-(5.45).

Otherwise, the calculation is very similar to the corresponding continuum Coulomb gauge calculation. Cf. V.N. Gribov, lecture at the 12th Winter School of the Leningrad Nuclear Physics Institute (1977),

S.D. Drell, in A Festschrift for Maurice Goldhaber, edited by G. Feinberg, A.W. Sunyar and J. Weneser (New York, New York Academy of Sciences, 1980) and T. D. Lee, Particle Physics and Introduction to Field Theory, (Harwood Academic Publishers, 1981).

10. G. N. Watson, Q.J.Math. 10, 266 (1939). 


\section{FIGURE CAPTIONS}

Figure 1. Equations (3.3)-(3.5) give an electromagnetic field configuration in the time-axial gauge within the unit cell in which $x, y, z$ all lie between 0 and $\ell$.

Figure 2. A picture of the localized QCD gauge field configuration (4.17) in the Coulomb gauge. All plaquettes are located on the $(x, y)$ plane at $z=0$. Move along the arrow direction of the central plaquette: Start from its lower left lattice site and label it 0 ; then label consecutively the other three lattice sites 1,2 and 3. The gluon field described by (4.17) corresponds to a link-flux distribution (in the notation of (4.14)) $a_{0}^{1}=b_{1}^{1}=-a_{3}^{1}=-b_{0}^{1}=1$ and all other $a_{j}^{\ell}, b_{j}^{\ell}$ and $c_{j}^{\ell}$ zero. Thus, Kirchhoff's law (4.15) is satisfied. The number inside each plaquette denotes the integral of $\ell^{-1} \int B_{j}^{1} d x d y$ over the plaquette. On account of the factor $\Delta(z)$, the gauge field distribution (4.17) is nonzero over two layers of 9 cubic cells each (making a total of 18 cells), extending from the 9 plaquettes at $z=0$ in the figure to $z= \pm \ell$.

Figure 3. The lattice sites $j, j^{\prime}, j^{\prime \prime}$ and $j^{\prime \prime \prime}$ are defined by (3.18). (a) shows the plaquettes $X_{j}, Y_{j}$ and $Z_{j}$ that are perpendicular to the $x, y$ and $z$ axes. (b) gives the plaquette-fluxes $\xi_{j}^{\ell}, \eta_{j}^{\ell}$ and $\zeta_{j}^{\ell}$ associated with these plaquettes. 